\title{
Unsteady analytical solutions of the spherical shallow water equations
}

\author{
Matthias Läuter, Dörthe Handorf, Klaus Dethloff \\ Alfred Wegener Institute Foundation for Polar and Marine Research \\ Post Box 6001 49, 14401 Potsdam, Germany
}

\begin{abstract}
A new class of unsteady analytical solutions of the spherical shallow water equations (SSWE) is presented. Analytical solutions of the SSWE are fundamental for the validation of barotropic atmospheric models. To date, only steady state analytical solutions are known from the literature. The unsteady analytical solutions of the SSWE are derived by applying the transformation method to the transition from a fixed cartesian to a rotating coordinate system. Fundamental examples of the new unsteady analytical solutions are presented for specific wind profiles. With the presented unsteady analytical solutions one can provide a measure of the numerical convergence in the case of a temporally evolving system. An application to the atmospheric model PLASMA shows the benefit of unsteady analytical solutions for the quantification of convergence properties.
\end{abstract}

Key words: shallow water equations, analytical solutions, atmospheric flow MSC: 35C05, 35Q30, 65Z05, 76N15

PACS: 47.40.Dc, 92.60.Bh

\section{Introduction}

During the course of the development of dynamical cores in numerical weather prediction and climate modelling the development of a global barotropic model for atmospheric flows is a crucial step. For this reason, in the past barotropic models have been developed which are based on the spherical shallow water equations (SSWE). The SSWE comprise the essential physical phenomena that are included in the full set of primitive equations, e. g. large scale planetary waves and gravity waves. Furthermore, the SSWE present the major difficulties found in the horizontal discretization of the 3-dimensional primitive equations. In order to validate implemented numerical methods for the SSWE, reasonable test cases are required.

To date, the comprehensive test suite in Williamson et al. (1992) is the common basis for newly developed global shallow water models. Succeeding, additional tests have been proposed in the literature, e. g. Galewsky et al. (2004). In the following, an overview of the known test cases is given which can be divided into four categories:

(1) Integrations of analytical solutions of simplified SSWE

Applying the common scale analysis, i. e. neglecting terms with small magnitude, simplified SSWE can be derived, like e.g. the linearized nondivergent barotropic vorticity equation or the geostrophic balance equation. Even though solutions of these systems are no analytical solutions of the full nonlinear SSWE, integrations

Published in J. Comput. Phys. (2005), 210, 535-553 
initialized by such initial states validate a SSWE model with respect to stability and noise generation.

For the first time Phillips (1959) proposed Rossby-Haurwitz waves as initial fields for integrations of a SSWE model. Rossby-Haurwitz waves are solutions of the linearized nondivergent barotropic vorticity equation and move from west to east without change of their shape. Since Hoskins (1973) showed that only Rossby-Haurwitz waves of zonal wavenumber 5 and less are stable, in the literature wavenumber 4 is applied to SSWE models, see e.g. Williamson et al. (1992), Jakob-Chien et al. (1995). McDonald and Bates (1989) proposed a geostrophic balanced initial state of zonal wavenumber 1 . Due to the presence of divergence the wave rotates clockwise around the pole, see e.g. Giraldo (2001).

(2) Integrations of steady state analytical solutions of the SSWE

Steady state analytical solutions of the SSWE mainly describe a purely zonal global flow with a balanced geopotential field. To our knowledge, solid body rotations on a nonrotating sphere have been described firstly by Dey (1969) and on a rotating sphere by Umscheid and Sankar-Rao (1971). Taking into account that, except for the Coriolis term, the SSWE are invariant under a rotation of the spherical coordinates, an inclined solid body rotation with an inclination angle $\alpha=\frac{\pi}{2}$ was given in Umscheid and Sankar-Rao (1971) and for arbitrary $\alpha \in[0, \pi]$ in Williamson and Browning (1973). Considering a more realistic zonal wind field, resembling a typical tropospheric jet, Browning et al. (1989) deduced a steady state analytical solution with compact support. Note that the balanced geopotential field is obtained by numerical integration. The mentioned solutions are inserted in Williamson et al. (1992, tests 2,3).

(3) Integrations of unsteady analytical solutions of the SSWE with the prescribedsolution forcing technique

For this class of solutions, the basic idea is to prescribe the exact solution of a nonlinear flow by adding approprate forcing terms to the SSWE. E. g. Rossby-Haurwitz waves (Merilees et al.; 1977), a linear combination of Hough-harmonics (Dee and Da Silva; 1986) or a translating low pressure center superimposed on a jet stream (Browning et al.; 1989; Williamson et al.; 1992) were applied as prescribed solutions.

(4) Integrations initialized by close to reality data fields

Analytical solutions of simplified SSWE or the full nonlinear SSWE, mentioned in (1) - (3), are not sufficient for the validation of SSWE models applied to more realistic flow situations. Instead, more elaborate initial states were used for SSWE models which describe real atmospheric phenomena, like e. g. the generation of planetary waves. E. g. Takacs (1988) introduced the initial state of a solid body rotation with a balanced geopotential corresponding to the global zonal geostrophic flow in Williamson et al. (1992, test 2), disturbed by an isolated mountain. Galewsky et al. (2004) proposed a balanced zonal wind field with a given meridional wind profile similar to Williamson et al. (1992, test 3), disturbed by a given initial perturbation of the balanced geopotential field. In both cases, the perturbations to the barotropic zonal flow induce planetary waves. Williamson et al. (1992, test 7) described inte- 
grations of several initial states which are derived from observational analyses of the $500 \mathrm{hPa}$ height and wind field.

This overview on tests for the SSWE reveals that analytical solutions of the SSWE are rare and especially unsteady analytical solutions are not available in the literature.

The quantification of the model's convergence properties is one of the main tasks of model validation. Reference solutions for a few non-analytical solutions were usually obtained with very high resolution spectral models, e. g. Jakob-Chien et al. (1995). These numerical solutions suffer from inaccuracies and thus allows only a comparison of model results. Additionally, quantitative information about a model can be obtained by analyzing conservation properties, but this does not necessarily provide information about convergence properties. Finally, the distance of an analytical and a numerical solution yields an objective measure of the quality of the model result. For that reason, the knowledge about analytical solutions is crucial for convergence studies and thus model validation.

Here, the transformation method for the SSWE is introduced and applied to provide unsteady analytical solutions for the validation process of atmospheric models. This method is based on the transformation from a fixed cartesian to a rotating coordinate system. Fundamental examples for unsteady analytical solutions of the SSWE are derived which have not been available in the literature until now. The benefit of the new analytical solutions for the validation of numerical models is demonstrated. Due to the limited complexity of the known analytical solutions, the physical relevance of the presented examples is limited, too. However, Piani and Norton (2002) showed that the simple solid body rotation is close to observed atmospheric data in the summer stratosphere.

To support a reliable formulation of the analytical solutions, two common formulations of the SSWE, namely in a cartesian coordinate system and in spherical coordinates are given in section 2. In section 3 we present the transformation method for the SSWE. By applying the transformation method we derive fundamental examples for unsteady analytical solutions in cartesian and spherical coordinates in section 4. Finally, we demonstrate the benefit of the new analytical solutions by analyzing the convergence properties of the barotropic model PLASMA (Parallel Large Scale Model of the Atmosphere) in section 5.

\section{Spherical shallow water equations}

In spherical geometry, the description of functions depends on the choice of local coordinates. Because the SSWE are formulated as well in cartesian as in spherical coordinates in the literature, both formulations are described in the following. The examples of unsteady analytical solutions of the SSWE in both formulations are given in section 4 .

We introduce some notation for preparation. Let $a=6.371 \cdot 10^{6} \mathrm{~m}$ be the Earth 
radius, $\Omega=7.292 \cdot 10^{-5} \mathrm{~s}^{-1}$ the Earth's angular velocity and $I=(0, T) \subset \mathbb{R}$ a fixed time intervall. For two vectors $\mathbf{x}, \mathbf{y} \in \mathbb{R}^{3}$ we denote the scalar product by $\mathbf{x} \cdot \mathbf{y}$. The components of a vector $\mathbf{x} \in \mathbb{R}^{3}$ with respect to the generic basis in Eq. (25) are denoted by $x_{i}=\mathbf{x} \cdot \mathbf{e}_{i}$ for $i=1,2,3$. Throughout the whole article we use the Einstein summation convention. I. e. if an index value, e.g. $i$, appears more than once in a term, e.g. in $A(i) B(i)$, and $i$ is not fixed outside of $A(i) B(i)$, then the reader has to substitude $A(i) B(i)$ by $\sum_{i=1}^{3} A(i) B(i)$.

\subsection{Representation in a cartesian coordinate system}

The representation of the SSWE in a cartesian coordinate system is based on the embedding of the sphere into $\mathbb{R}^{3}$, see e. g. Côté (1988); Williamson et al. (1992); Swarztrauber et al. (1997); Stuhne and Peltier (1999); Tomita et al. (2001); Heinze and Hense (2002); Ringler and Randall (2002); Giraldo and Warburton (2005); Bonaventura and Ringler (2004). Thus, the prognostic functions as well as the differential operators are defined in $\mathbb{R}^{3}$.

We define the spherical shell with the thickness $2 \varepsilon>0$

$$
G_{\varepsilon}=\left\{\mathbf{x} \in \mathbb{R}^{3}|a-\varepsilon<| \mathbf{x} \mid<a+\varepsilon\right\}
$$

and the sphere $S=\left\{\mathbf{x} \in \mathbb{R}^{3}|| \mathbf{x} \mid=a\right\}$ as spatial domains. The velocity field $\mathbf{u}: G_{\varepsilon} \times I \rightarrow \mathbb{R}^{3}$ and the geopotential field $\Phi: G_{\varepsilon} \times I \rightarrow \mathbb{R}$ are smooth functions in $G_{\varepsilon}$. We call $(\mathbf{u}, \Phi)$ a solution of the SSWE in the cartesian coordinate system, if $\mathbf{u}$ and $\Phi$ fulfill the equations

$$
\begin{aligned}
\partial_{t} \mathbf{u}+\mathbf{u} \cdot \nabla_{S} \mathbf{u}+\nabla_{S} \Phi & =-f \mathbf{n} \times \mathbf{u}-\frac{|\mathbf{u}|^{2}}{|\mathbf{x}|} \mathbf{n}, \\
\partial_{t} \Phi+\mathbf{u} \cdot \nabla_{S}\left(\Phi-\Phi_{B}\right)+\left(\Phi-\Phi_{B}\right) \operatorname{div}_{S} \mathbf{u} & =0 \\
\mathbf{u} \cdot \mathbf{n} & =0
\end{aligned}
$$

in the space-time domain $S \times I$. Here $\mathbf{n}=\frac{\mathbf{x}}{|\mathbf{x}|}$ is the normalized radial vector, $\nabla_{S} g=\nabla g-(\mathbf{n} \cdot \nabla g) \mathbf{n}$ the horizontal gradient, $\operatorname{div}_{S} \mathbf{v}=\operatorname{div}(\mathbf{v}-\mathbf{v} \cdot \mathbf{n} \mathbf{n})$ the horizontal divergence, $f=2 \Omega \mathbf{e}_{3} \cdot \mathbf{n}$ the Coriolis parameter and $\Phi_{B}: G_{\varepsilon} \rightarrow \mathbb{R}$ the given orography field. The functions $\mathbf{u}$ and $\Phi$ have to be defined in a neighborhood of $S$, here $G_{\varepsilon}$, in order to ensure well defined differential operators $\operatorname{div}_{S} \mathbf{u}$ and $\nabla_{S} \Phi$. In any case, since the values of all terms in the Eqs. (2) - (4) are independent on the function values of $\mathbf{u}$ and $\Phi$ in $G_{\varepsilon} \backslash S$, the temporal evolution of $(\mathbf{u}, \Phi)$ in $S$ depends on the initial conditions in $S$ only. Thus, a function $\left(\left.\mathbf{u}\right|_{S},\left.\Phi\right|_{S}\right)$ which is defined in $S$ only, is a solution of the SSWE, if any smooth continuation $(\mathbf{u}, \Phi)$ to $G_{\varepsilon}$ solves the Eqs. (2) - (4).

\subsection{Representation in spherical coordinates}

An alternative formulation of the SSWE (2) - (4) in spherical coordinates is described by e.g. Côté and Staniforth (1990); Williamson et al. (1992); Jakob-Chien 
et al. (1995); Lin and Rood (1997); Tolstykh (2002); Jablonowski (2004). Starting with a solution of the Eqs. (2) - (4), we obtain the SSWE in spherical coordinates for the zonal and meridional velocities and the geopotential field.

The spherical coordinates are defined in $\Sigma_{\varepsilon}=(0,2 \pi) \times\left(-\frac{\pi}{2}, \frac{\pi}{2}\right) \times(a-\varepsilon, a+\varepsilon)$ by the position vector

$$
\mathbf{r}: \Sigma_{\varepsilon} \rightarrow \mathbf{r}\left(\Sigma_{\varepsilon}\right), \quad \mathbf{r}(\lambda, \theta, r)=r(\cos \lambda \cos \theta, \sin \lambda \cos \theta, \sin \theta)^{\mathrm{T}} .
$$

We define the local cartesian coordinate system $(\mathbf{i}(\mathbf{x}), \mathbf{j}(\mathbf{x}), \mathbf{n}(\mathbf{x}))$ at each spatial point $\mathbf{x} \in \mathbf{r}\left(\Sigma_{\varepsilon}\right)$ by

$$
\begin{aligned}
\mathbf{i}(\mathbf{x}) & =\frac{-x_{2} \mathbf{e}_{1}+x_{1} \mathbf{e}_{2}}{\sqrt{x_{1}^{2}+x_{2}^{2}}}, \quad \mathbf{j}(\mathbf{x})=\frac{-x_{1} x_{3} \mathbf{e}_{1}-x_{2} x_{3} \mathbf{e}_{2}+\left(x_{1}^{2}+x_{2}^{2}\right) \mathbf{e}_{3}}{|\mathbf{x}| \sqrt{x_{1}^{2}+x_{2}^{2}}}, \\
\mathbf{n}(\mathbf{x}) & =\frac{\mathbf{x}}{|\mathbf{x}|}
\end{aligned}
$$

$\left(\mathbf{e}_{1}, \mathbf{e}_{2}, \mathbf{e}_{3}\right)$ is the generic basis from Eq. (25). The vectors $\mathbf{i}, \mathbf{j}$ and $\mathbf{n}$ point to the eastward, the northward and the vertical direction, respectively.

Let $(\mathbf{u}, \Phi)$ be a solution of the SSWE in the cartesian coordinate system (2) - (4). We define the zonal, meridional and vertical velocity components $u, v, w: \Sigma_{\varepsilon} \times I \rightarrow \mathbb{R}$ by

$$
u(.)=\mathbf{u} \cdot \mathbf{i}(\mathbf{r}(.)), \quad v(.)=\mathbf{u} \cdot \mathbf{j}(\mathbf{r}(.)), \quad w(.)=\mathbf{u} \cdot \mathbf{n}(\mathbf{r}(.)) .
$$

The geopotential field $\Psi: \Sigma_{\varepsilon} \times I \rightarrow \mathbb{R}$ in spherical coordinates is defined by $\Psi()=$. $\Phi(\mathbf{r}()$.$) .$

To derive prognostic equations for $u, v$ and $w$ we use the scalar multiplication of Eq. (2) and the basis vectors $\mathbf{i}, \mathbf{j}$ and $\mathbf{n}$, respectively. We obtain

$$
\begin{aligned}
\frac{\partial u}{\partial t}+\frac{u}{r}\left(\frac{1}{\cos \theta} \frac{\partial u}{\partial \lambda}-v \tan \theta\right)+\frac{v}{r} \frac{\partial u}{\partial \theta}+\frac{u w}{r}+\frac{1}{r \cos \theta} \frac{\partial \Psi}{\partial \lambda}-f v & =0, \\
\frac{\partial v}{\partial t}+\frac{u}{r}\left(\frac{1}{\cos \theta} \frac{\partial v}{\partial \lambda}+u \tan \theta\right)+\frac{v}{r} \frac{\partial v}{\partial \theta}+\frac{v w}{r}+\frac{1}{r} \frac{\partial \Psi}{\partial \theta}+f u & =0, \\
\frac{\partial w}{\partial t}+\frac{u}{r \cos \theta} \frac{\partial w}{\partial \lambda}+\frac{v}{r} \frac{\partial w}{\partial \theta}-\frac{u^{2}+v^{2}}{r}+\frac{u^{2}+v^{2}}{r} & =0 \\
\frac{\partial \Psi}{\partial t}+\frac{u}{r \cos \theta} \frac{\partial \Psi-\Psi_{B}}{\partial \lambda}+\frac{v}{r} \frac{\partial \Psi-\Psi_{B}}{\partial \theta}+\frac{\Psi-\Psi_{B}}{r \cos \theta}\left(\frac{\partial u}{\partial \lambda}+\frac{\partial(v \cos \theta)}{\partial \theta}\right) & =0, \\
w & =0
\end{aligned}
$$

in the spatial domain $(0,2 \pi) \times\left(-\frac{\pi}{2}, \frac{\pi}{2}\right) \times\{a\}$. The prognostic equation for $\Psi$ is a consequence of Eq. (3). By omitting the vertical velocity component $w$ this system simplifies in $(0,2 \pi) \times\left(-\frac{\pi}{2}, \frac{\pi}{2}\right) \times\{a\}$ to

$$
\begin{array}{r}
\frac{\partial u}{\partial t}+\frac{u}{a}\left(\frac{1}{\cos \theta} \frac{\partial u}{\partial \lambda}-v \tan \theta\right)+\frac{v}{a} \frac{\partial u}{\partial \theta}+\frac{1}{a \cos \theta} \frac{\partial \Psi}{\partial \lambda}-f v=0, \\
\frac{\partial v}{\partial t}+\frac{u}{a}\left(\frac{1}{\cos \theta} \frac{\partial v}{\partial \lambda}+u \tan \theta\right)+\frac{v}{a} \frac{\partial v}{\partial \theta}+\frac{1}{a} \frac{\partial \Psi}{\partial \theta}+f u=0, \\
\frac{\partial \Psi}{\partial t}+\frac{u}{a \cos \theta} \frac{\partial \Psi-\Psi_{B}}{\partial \lambda}+\frac{v}{a} \frac{\partial \Psi-\Psi_{B}}{\partial \theta}+\frac{\Psi-\Psi_{B}}{a \cos \theta}\left(\frac{\partial u}{\partial \lambda}+\frac{\partial(v \cos \theta)}{\partial \theta}\right)=0 .
\end{array}
$$


These equations are called the SSWE in spherical coordinates.

\section{The transformation method}

The transformation method for the SSWE consists of the transition from a fixed coordinate system to a rotating coordinate system. This method is well known from the literature, e. g. (Haltiner and Williams; 1980; Pedlosky; 1987; Dutton; 1995) for equations in $\mathbb{R}^{3}$. Due to the embedding of the sphere $S$ in $\mathbb{R}^{3}$, the method leads to a transformation for equations on $S$. Practically, the transformation method adds an angular velocity to the original velocity field and generates the Coriolis term in the dynamical equations.

In a fixed coordinate system we consider smooth functions $\mathbf{v}: G_{\varepsilon} \times I \rightarrow \mathbb{R}^{3}$ and $\Psi: G_{\varepsilon} \times I \rightarrow \mathbb{R}$ in the spatial domain $G_{\varepsilon}$, defined in Eq. (1). We call the system

$$
\begin{aligned}
\partial_{t} \mathbf{v}+\mathbf{v} \cdot \nabla_{S} \mathbf{v}+\nabla_{S} \Psi & =-\frac{|\mathbf{v}|^{2}}{|\mathbf{x}|} \mathbf{n}, \\
\partial_{t}\left(\Psi-\Psi_{B}\right)+\mathbf{v} \cdot \nabla_{S}\left(\Psi-\Psi_{B}\right)+\left(\Psi-\Psi_{B}\right) \operatorname{div}_{S} \mathbf{v} & =0 \\
\mathbf{v} \cdot \mathbf{n} & =0
\end{aligned}
$$

in $S$ the SSWE without Coriolis force, where $\Psi_{B}: G_{\varepsilon} \times I \rightarrow \mathbb{R}$ is a given time dependent orography field.

Remark 1 Because the Earth rotates in the inertial system of (8) - (10), it is natural to consider the orography $\Psi_{B}$ as a time dependent function.

\subsection{Rotating coordinate system}

The transition from a fixed to a rotating coordinate system can be obtained by applying general curvilinear coordinates, see appendix A. For a velocity field $\mathbf{v}$ and a scalar field $\Psi$ in the fixed coordinate system we consider the fields $\mathbf{u}$ and $\Phi$ in the rotating coordinate system. We give the well known transformation of their total derivatives.

We consider the generic basis $\left(\mathbf{e}_{1}, \mathbf{e}_{2}, \mathbf{e}_{3}\right)$ from Eq. (25) as the basis of the fixed coordinate system. For all $t \in I$ we define the basis functions of the rotating coordinate system by

$$
\begin{aligned}
& \mathbf{b}_{1}(t)=\cos (\Omega t) \mathbf{e}_{1}+\sin (\Omega t) \mathbf{e}_{2} \\
& \mathbf{b}_{2}(t)=-\sin (\Omega t) \mathbf{e}_{1}+\cos (\Omega t) \mathbf{e}_{2} \\
& \mathbf{b}_{3}(t)=\mathbf{e}_{3}
\end{aligned}
$$

By applying the equations of appendix A we determine the transition of coordinates in the spatial domain $G_{\varepsilon}$ through the coordinate transformation function $\varphi_{t}: G_{\varepsilon} \rightarrow$ 
$G_{\varepsilon}$. For all $\mathbf{x}, \mathbf{y} \in G_{\varepsilon}$ we define $\varphi_{t}$, its inverse $\varphi_{t}^{-1}: G_{\varepsilon} \rightarrow G_{\varepsilon}$ and the position vector $\mathbf{r}: G_{\varepsilon} \times I \rightarrow G_{\varepsilon}$ by

$$
\varphi_{t}(\mathbf{x})=\mathbf{x} \cdot \mathbf{b}_{i} \mathbf{e}_{i}, \quad \mathbf{r}(\mathbf{y}, t)=\varphi_{t}^{-1}(\mathbf{y})=\mathbf{y} \cdot \mathbf{e}_{i} \mathbf{b}_{i} .
$$

Then the relation

$$
\mathbf{b}_{i}(t)=\frac{\partial \mathbf{r}}{\partial y_{i}}(t)=\frac{\partial \varphi_{t, i}}{\partial x_{j}} \mathbf{e}_{j}, \quad \text { for } i=1,2,3
$$

holds. With $\Omega=\Omega \mathbf{e}_{3}$ and Eq. (26) for the velocity of a particle which is resting in the rotating coordinate system we obtain the equation

$$
\mathbf{w}(\mathbf{x})=\Omega\left(-\mathbf{r} \cdot \mathbf{e}_{2} \mathbf{e}_{1}+\mathbf{r} \cdot \mathbf{e}_{1} \mathbf{e}_{2}\right)=\Omega \mathbf{e}_{3} \times \mathbf{r}\left(\varphi_{t}(\mathbf{x}), t\right)=\Omega \times \mathbf{x} .
$$

Let now $\mathbf{v}: G_{\varepsilon} \rightarrow \mathbb{R}^{3}$ and $\Psi: G_{\varepsilon} \rightarrow \mathbb{R}$ be smooth fields, describing the motion of a fluid in the fixed coordinate system. The same physical flow is described by the fields $\mathbf{u}: G_{\varepsilon} \rightarrow \mathbb{R}^{3}$ and $\Phi: G_{\varepsilon} \rightarrow \mathbb{R}$ in the rotating coordinate system at a fixed time $t \in I$. The relation between the corresponding fields follows from the Eqs. (27) and (12) and we obtain for all $\mathrm{x} \in G_{\varepsilon}$ the equations

$$
\mathbf{v}(\mathbf{x})=\left.\mathbf{u}\right|_{\varphi_{t}(\mathbf{x})} \cdot \mathbf{e}_{i} \mathbf{b}_{i}+\Omega \times \mathbf{x}, \quad \Psi(\mathbf{x})=\left.\Phi\right|_{\varphi_{t}(\mathbf{x})} .
$$

The last equation provides the transformation from the fixed to the rotating coordinate system using the transformation function $\varphi_{t}$ and the rotation operator $\cdot \mathbf{e}_{i} \mathbf{b}_{i}$. This is equivalent to the transformations in (Haltiner and Williams; 1980; Pedlosky; 1987; Dutton; 1995) where appropriate indices for vectors in the different coordinate systems are used. We recall the transformation of the total derivatives for $\mathbf{v}$ and $\Psi$, see e.g. (Haltiner and Williams; 1980; Pedlosky; 1987; Dutton; 1995; Läuter; 2004).

Theorem 1 Let $\mathbf{v}: G_{\varepsilon} \times I \rightarrow \mathbb{R}^{3}$ and $\Psi: G_{\varepsilon} \times I \rightarrow \mathbb{R}$ be smooth fields in the fixed coordinate system. By Eq. (13) we define the velocity field $\mathbf{u}: G_{\varepsilon} \times I \rightarrow \mathbb{R}^{3}$ and the scalar field $\Phi: G_{\varepsilon} \times I \rightarrow \mathbb{R}$ in the rotating coordinate system. Then the following properties hold for the total derivatives of $\mathbf{u}$ and $\Phi$ in $G_{\varepsilon} \times I$ :

$$
\begin{aligned}
{\left[\partial_{t} \mathbf{v}+\mathbf{v} \cdot \nabla \mathbf{v}\right]_{\mathbf{x}} } & =\left[\partial_{t} \mathbf{u}+\mathbf{u} \cdot \nabla \mathbf{u}+2 \boldsymbol{\Omega} \times \mathbf{u}+\boldsymbol{\Omega} \times(\boldsymbol{\Omega} \times \mathbf{y})\right]_{\mathbf{y}=\varphi_{t}(\mathbf{x})} \cdot \mathbf{e}_{i} \mathbf{b}_{i}, \\
{\left[\partial_{t} \Psi+\mathbf{v} \cdot \nabla \Psi\right]_{\mathbf{x}} } & =\left[\partial_{t} \Phi+\mathbf{u} \cdot \nabla \Phi\right]_{\varphi_{t}(\mathbf{x})} .
\end{aligned}
$$

\subsection{Transformation of the shallow water equations}

Theorem 1 includes the transformation of the total derivative from the fixed to the rotating coordinate system. To obtain a transformation of the whole SSWE without Coriolis force in terms of $\mathbf{v}$ and $\Psi$, we have to specify the transformation for the remaining terms in the Eqs. (8) - (10). This results in the SSWE in terms of $\mathbf{u}$ and $\Phi$ in the rotating coordinate system.

For the transformation of the centrifugal force term $\frac{|\mathbf{v}|^{2}}{|\mathbf{x}|} \mathbf{n}$ in Eq. (8) we substitute $\mathbf{v}$ by $\mathbf{u}$ with Eq. (13) which leads with Eq. (15) to a decomposition of the centrifugal 
force. A fraction of that centrifugal force is expressible as a gradient field of the auxiliary geopotential field

$$
\Psi_{Z}(\mathbf{x})=-\frac{(\Omega \cdot \mathbf{x})^{2}}{2}, \quad \forall \mathbf{x} \in \mathbb{R}^{3} .
$$

We will take advantage of this property at the end of the proof to Theorem 3 and provide the required arithmetics in Lemma 2.

Lemma 2 For all vectors $\mathbf{u}, \mathbf{y} \in \mathbb{R}^{3}$ with $\mathbf{y} \neq 0$ the equations

$$
\begin{aligned}
\frac{|\mathbf{u}+\boldsymbol{\Omega} \times \mathbf{y}|^{2}}{|\mathbf{y}|} & =\frac{|\mathbf{u}|^{2}}{|\mathbf{y}|}+2 \mathbf{u} \cdot(\boldsymbol{\Omega} \times \mathbf{n})+(\boldsymbol{\Omega} \times \mathbf{n}) \cdot(\boldsymbol{\Omega} \times \mathbf{y}), \\
-\nabla_{S} \Psi_{Z}(\mathbf{y}) & =\boldsymbol{\Omega} \times(\boldsymbol{\Omega} \times \mathbf{y})-\boldsymbol{\Omega} \times(\boldsymbol{\Omega} \times \mathbf{y}) \cdot \mathbf{n} \mathbf{n}
\end{aligned}
$$

hold. If additionally $\mathbf{u} \cdot \mathbf{n}(\mathbf{y})=0$ holds, it follows

$$
\boldsymbol{\Omega} \times \mathbf{u}-(\boldsymbol{\Omega} \times \mathbf{u}) \cdot \mathbf{n} \mathbf{n}=\boldsymbol{\Omega} \cdot \mathbf{n} \mathbf{n} \times \mathbf{u} .
$$

Proof:

For $\mathbf{u}, \mathbf{y} \in \mathbb{R}^{3}$ with $\mathbf{y} \neq 0$ and $\mathbf{a}, \mathbf{b}, \mathbf{c} \in \mathbb{R}^{3}$ with $|\mathbf{c}|=1$ some arithmetics yield

$$
\begin{aligned}
|\mathbf{u}+\boldsymbol{\Omega} \times \mathbf{y}|^{2} & =|\mathbf{u}|^{2}+2 \mathbf{u} \cdot(\boldsymbol{\Omega} \times \mathbf{y})+(\boldsymbol{\Omega} \times \mathbf{y}) \cdot(\boldsymbol{\Omega} \times \mathbf{y}) \\
-\nabla_{S} \Psi_{Z}(\mathbf{y}) & =\frac{\Omega^{2} y_{3}}{|\mathbf{y}|^{2}}\left(-y_{1} y_{3},-y_{2} y_{3}, y_{1}^{2}+y_{2}^{2}\right)^{\mathrm{T}}, \\
(\mathbf{a} \times \mathbf{b}) \cdot \mathbf{c} \mathbf{c} & =(\mathbf{a}-\mathbf{a} \cdot \mathbf{c} \mathbf{c}) \times(\mathbf{b}-\mathbf{b} \cdot \mathbf{c} \mathbf{c}) .
\end{aligned}
$$

Whereas Eqs. (15) and (16) are consequences of the first two equations, Eq. (17) follows from the third equation with $\mathbf{u} \cdot \mathbf{n}(\mathbf{y})=0, \mathbf{a}=\boldsymbol{\Omega}, \mathbf{b}=\mathbf{u}$ and $\mathbf{c}=\mathbf{n}(\mathbf{y})$.

Now we are prepared to give the main Theorem which states the relationship between a solution $(\mathbf{v}, \Psi)$ of the SSWE without Coriolis force and the corresponding function $(\mathbf{u}, \Phi)$ in the rotating coordinate system.

Theorem 3 Let $(\mathbf{v}, \Psi)$ be a solution of the SSWE without Coriolis force (8) - (10) with the time dependent orography $\Psi_{B}: G_{\varepsilon} \times I \rightarrow \mathbb{R}$. Further on let $\mathbf{u}: G_{\varepsilon} \times I \rightarrow \mathbb{R}^{3}$ and $\Phi, \Phi_{B}: G_{\varepsilon} \times I \rightarrow \mathbb{R}$ be the functions in the rotating coordinate system which fulfill the equations

$$
\begin{aligned}
\mathbf{u}(\mathbf{y}) & =[\mathbf{v}-\Omega \times \mathbf{x}]_{\mathbf{x}=\varphi_{t}^{-1}(\mathbf{y})} \cdot \mathbf{b}_{i} \mathbf{e}_{i}, \\
\Phi(\mathbf{y}) & =\left[\Psi-\Psi_{Z}\right]_{\varphi_{t}^{-1}(\mathbf{y})}, \\
\Phi_{B}(\mathbf{y}) & =\left[\Psi_{B}-\Psi_{Z}\right]_{\varphi_{t}^{-1}(\mathbf{y})} .
\end{aligned}
$$

Then $(\mathbf{u}, \Phi)$ is a solution of the SSWE (2) - (4) with the orography $\Phi_{B}$. 
Proof:

For the geopotential field $\Psi_{Z}$ in Eq. (14) we define the corresponding field in the rotating coordinate system for $\mathbf{y} \in G_{\varepsilon}$ by $\Phi_{Z}(\mathbf{y})=\left.\Psi_{Z}\right|_{\varphi_{t}^{-1}(\mathbf{y})}=\Psi_{Z}(\mathbf{y})$. Let $\mathbf{v}, \mathbf{u}$ : $G_{\varepsilon} \times I \rightarrow \mathbb{R}^{3}$ and $\Psi, \Phi: G_{\varepsilon} \times I \rightarrow \mathbb{R}$ be functions with the properties in Theorem 3. Then for all $\mathbf{x}, \mathbf{y} \in G_{\varepsilon}$ it follows

$$
\nabla \Psi(\mathbf{x})=\frac{\partial \Psi}{\partial x_{i}} \mathbf{e}_{i}, \quad \nabla \Phi(\mathbf{y})=\frac{\partial \Phi}{\partial y_{i}} \mathbf{e}_{i}, \quad \mathbf{n}(\mathbf{x})=\left.\mathbf{n}\right|_{\varphi_{t}(\mathbf{x})} \cdot \mathbf{e}_{i} \mathbf{b}_{i} .
$$

Applying this and Eq. (12), the transformation of the terms in the Eqs. (8) - (10) for all $\mathbf{x} \in G_{\varepsilon}$ leads to

$$
\begin{aligned}
\nabla_{S} \Psi(\mathbf{x}) & =\frac{\partial\left(\Phi+\Phi_{Z}\right)}{\partial y_{i}} \frac{\partial \varphi_{t, i}}{\partial x_{j}}\left(\mathbf{e}_{j}-\mathbf{e}_{j} \cdot \mathbf{n} \mathbf{n}\right) \\
& =\frac{\partial\left(\Phi+\Phi_{Z}\right)}{\partial y_{i}} \mathbf{b}_{i} \cdot \mathbf{e}_{j}\left(\mathbf{e}_{j}-\mathbf{e}_{j} \cdot \mathbf{n} \mathbf{n}\right) \\
& =\nabla\left(\Phi+\Phi_{Z}\right) \cdot \mathbf{e}_{i}\left(\mathbf{b}_{i}-\mathbf{b}_{i} \cdot \mathbf{n}(\mathbf{x}) \mathbf{n}(\mathbf{x})\right) \\
& =\nabla\left(\Phi+\Phi_{Z}\right) \cdot \mathbf{e}_{i}\left[\mathbf{b}_{i}-\left(\mathbf{e}_{i} \cdot \mathbf{n}\right)\left(\mathbf{n} \cdot \mathbf{e}_{j}\right) \mathbf{b}_{j}\right]_{\varphi_{t}(\mathbf{x})} \\
& =\left.\nabla_{S}\left(\Phi+\Phi_{Z}\right)\right|_{\varphi_{t}(\mathbf{x})} \cdot \mathbf{e}_{i} \mathbf{b}_{i}, \\
\frac{|\mathbf{v}(\mathbf{x})|^{2}}{|\mathbf{x}|} & \mathbf{n}(\mathbf{x})=\left[\frac{|\mathbf{u}+\mathbf{\Omega} \times \mathbf{y}|^{2}}{|\mathbf{y}|} \mathbf{n}\right]_{\mathbf{y}=\varphi_{t}(\mathbf{x})} \cdot \mathbf{e}_{i} \mathbf{b}_{i}
\end{aligned}
$$

and

$$
\begin{aligned}
\operatorname{div}_{S} \mathbf{v}(\mathbf{x}) & =\frac{\partial(\mathbf{v}-\mathbf{v} \cdot \mathbf{n} \mathbf{n}) \cdot \mathbf{e}_{j}}{\partial x_{j}}=\left.\frac{\partial(\mathbf{v}-\mathbf{v} \cdot \mathbf{n} \mathbf{n}) \circ \varphi_{t}^{-1}}{\partial y_{i}}\right|_{\varphi_{t}(\mathbf{x})} \cdot \mathbf{e}_{j} \frac{\partial \varphi_{t, i}}{\partial x_{j}} \\
& =\left.\frac{\partial \mathbf{u}+\mathbf{\Omega} \times \mathbf{y}-\mathbf{u} \cdot \mathbf{n} \mathbf{n}}{\partial y_{i}}\right|_{\mathbf{y}=\varphi_{t}(\mathbf{x})} \cdot \mathbf{e}_{j} \mathbf{b}_{j} \cdot \mathbf{b}_{i} \\
& =\left.\frac{\partial \mathbf{u}-\mathbf{u} \cdot \mathbf{n} \mathbf{n}}{\partial y_{i}}\right|_{\varphi_{t}(\mathbf{x})} \cdot \mathbf{e}_{i}=\left.\operatorname{div}_{S} \mathbf{u}\right|_{\varphi_{t}(\mathbf{x})} \cdot
\end{aligned}
$$

Applying Theorem 1 and Eqs. (18) - (20) to the Eqs. (8) - (10), we obtain

$$
\begin{aligned}
\partial_{t} \mathbf{u}+\mathbf{u} \cdot \nabla_{S} \mathbf{u}+2 \boldsymbol{\Omega} \times \mathbf{u}+\boldsymbol{\Omega} \times(\boldsymbol{\Omega} \times \mathbf{y})+\nabla_{S}\left(\Phi+\Phi_{Z}\right) & =-\frac{|\mathbf{u}+\boldsymbol{\Omega} \times \mathbf{y}|^{2}}{|\mathbf{y}|} \mathbf{n} \\
\partial_{t}\left(\Phi-\Phi_{B}\right)+\mathbf{u} \cdot \nabla_{S}\left(\Phi-\Phi_{B}\right)+\left(\Phi-\Phi_{B}\right) \operatorname{div}_{S} \mathbf{u} & =0 \\
\mathbf{u} \cdot \mathbf{n} & =0
\end{aligned}
$$

for all $(\mathbf{y}, t) \in S \times I$. By using Lemma 2 we find that $(\mathbf{u}, \Phi)$ fulfills the SSWE $(2)-$ (4) which completes the proof.

According to remark 1, it was natural to consider the time dependent orography $\Psi_{B}$. In contrast to that, the time dependence of $\Phi_{B}$ has to vanish for physically relevant solutions of Theorem 3 , because the rotating coordinate system $\left(\mathbf{b}_{1}, \mathbf{b}_{2}, \mathbf{b}_{3}\right)$ is fixed on the rotating Earth. 


\section{Analytical solutions}

The transformation method in section 3 allows us to derive a solution of the SSWE $(2)-(4)$, if a solution of the SSWE without Coriolis force (8) - (10) is known. The results are unsteady solutions of the SSWE with a time period of 1 day. In the following, we derive 3 examples of unsteady solutions of the SSWE, an unsteady axially symmetric solution with an arbitrary wind profile and two special cases of it. The first is an unsteady solid body rotation and the second an unsteady solid body rotation with a superimposed unsteady jet stream.

\subsection{A steady state solution}

To apply Theorem 3 a solution of the SSWE without Coriolis force (8) - (10) is required. We confine ourselves to an example of an axially symmetric solution of the SSWE, see e.g. (Browning et al.; 1989; Williamson et al.; 1992; Läuter; 2004).

Example 1 (Steady state axially symmetric flow) Let $\mathbf{b} \in \mathbb{R}^{3},|\mathbf{b}|=1$ be a fixed symmetry axis, $k_{1}, k_{2} \in \mathbb{R}$ arbitrary constants and $v_{p}:[-1,1] \rightarrow \mathbb{R}$ a smooth velocity profile with a bounded integral

$$
\int_{-\frac{\pi}{2}}^{\frac{\pi}{2}}\left|\tan \phi v_{p}^{2}(\sin \phi)\right| d \phi<+\infty .
$$

Then the function $(\mathbf{v}, \Psi)$, defined for $\mathbf{x} \in G_{\varepsilon}$

$$
\begin{aligned}
\mathbf{v}(\mathbf{x}) & =v_{p}(\mathbf{b} \cdot \mathbf{n}(\mathbf{x})) \frac{\mathbf{b} \times \mathbf{n}(\mathbf{x})}{|\mathbf{b} \times \mathbf{n}(\mathbf{x})|}, \\
\Psi(\mathbf{x}) & =-\int_{0}^{\arcsin (\mathbf{b} \cdot \mathbf{n}(\mathbf{x}))} \tan \phi v_{p}^{2}(\sin \phi) d \phi+k_{1}, \\
\Psi_{B}(\mathbf{x}) & =k_{2}
\end{aligned}
$$

is a solution of the SSWE without Coriolis force (8) - (10) with the orography $\Psi_{B}$.

Remark 2 To obtain a reasonable solution in example 1, that is to obtain a nonnegative mass in Eq. (9), the constants $k_{1}$ and $k_{2}$ should be chosen such, that $\Psi_{B}<\Psi$ holds.

A smooth bounded velocity profile $v_{p}$ with compact support in $(0,1)$ is sufficient to fulfill Eq. (21). 


\subsection{Unsteady solutions}

In this section we obtain unsteady solutions of the SSWE (2) - (4) by applying Theorem 3 to the example 1 of a steady state axially symmetric flow. Example 2 is the general case of an unsteady axially symmetric flow, see Läuter (2004, p. 69f). The examples 3 and 4 are special cases of example 2. All derived solutions describe a precession around the Earth's axis with a time period of 1 day. Because example 1 gives a nondivergent solution, the unsteady solutions are nondivergent, too.

Example 2 (Unsteady axially symmetric flow) Let $\mathbf{a} \in \mathbb{R}^{3},|\mathbf{a}|=1$ be a fixed symmetry axis, $k_{1}, k_{2} \in \mathbb{R}$ arbitrary constants and $u_{p}:[-1,1] \rightarrow \mathbb{R}$ a smooth velocity profile with a bounded integral $\int_{-\frac{\pi}{2}}^{\frac{\pi}{2}}\left|\tan \phi u_{p}^{2}(\sin \phi)\right| d \phi<+\infty$. Further on let $\varphi_{t}$ be the coordinate transformation function for the rotation in Eq. (11). Then the function $(\mathbf{u}, \Phi)$, defined for $(\mathbf{x}, t) \in G_{\varepsilon} \times I$

$$
\begin{aligned}
& \mathbf{u}(\mathbf{x}, t)=u_{p}\left(\varphi_{t}(\mathbf{a}) \cdot \mathbf{n}(\mathbf{x})\right) \frac{\varphi_{t}(\mathbf{a}) \times \mathbf{n}(\mathbf{x})}{\left|\varphi_{t}(\mathbf{a}) \times \mathbf{n}(\mathbf{x})\right|}-\mathbf{\Omega} \times \mathbf{x}, \\
& \Phi(\mathbf{x}, t)=-\int_{0}^{\arcsin \left(\varphi_{t}(\mathbf{a}) \cdot \mathbf{n}(\mathbf{x})\right)} \tan \phi u_{p}^{2}(\sin \phi) d \phi+\frac{(\boldsymbol{\Omega} \cdot \mathbf{x})^{2}}{2}+k_{1} \\
& \Phi_{B}(\mathbf{x})=\frac{(\boldsymbol{\Omega} \cdot \mathbf{x})^{2}}{2}+k_{2}
\end{aligned}
$$

is a solution of SSWE (2) - (4) with the orography $\Phi_{B}$. To prove this, we apply Theorem 3 to the functions $\mathbf{v}$ and $\Psi$ of example 1 with the parameters $\mathbf{b}=\mathbf{a}$ and $v_{p}=u_{p}$. The scalar fields vorticity $\zeta$ and divergence $\delta$ fulfill the equations

$$
\begin{aligned}
& \zeta(\mathbf{x}, t)=\frac{u_{p}\left(\varphi_{t}(\mathbf{a}) \cdot \mathbf{n}\right) \varphi_{t}(\mathbf{a}) \cdot \mathbf{n}}{a \sqrt{1-\left(\varphi_{t}(\mathbf{a}) \cdot \mathbf{n}\right)^{2}}}-\frac{u_{p}^{\prime}\left(\varphi_{t}(\mathbf{a}) \cdot \mathbf{n}\right)}{a} \sqrt{1-\left(\varphi_{t}(\mathbf{a}) \cdot \mathbf{n}\right)^{2}}-2 \boldsymbol{\Omega} \cdot \mathbf{n}, \\
& \delta(\mathbf{x}, t)=0 .
\end{aligned}
$$

With the Eqs. (5), (7) and the local coordinate system in Eq. (6) this solution may be written in spherical coordinates. We conclude, that for all $(\lambda, \theta, r) \in \Sigma_{\varepsilon}, t \in I$ and with $\mathbf{x}=\mathbf{r}(\lambda, \theta, r)$ the functions

$$
\begin{aligned}
u(\lambda, \theta, r, t) & =\frac{u_{p}\left(\varphi_{t}(\mathbf{a}) \cdot \mathbf{n}(\mathbf{x})\right)}{\left|\varphi_{t}(\mathbf{a}) \times \mathbf{n}(\mathbf{x})\right|} \varphi_{t}(\mathbf{a}) \cdot \mathbf{j}(\mathbf{x})-r \Omega \cos \theta, \\
v(\lambda, \theta, r, t) & =-\frac{u_{p}\left(\varphi_{t}(\mathbf{a}) \cdot \mathbf{n}(\mathbf{x})\right)}{\left|\varphi_{t}(\mathbf{a}) \times \mathbf{n}(\mathbf{x})\right|} \varphi_{t}(\mathbf{a}) \cdot \mathbf{i}(\mathbf{x}), \\
\Phi(\lambda, \theta, r, t) & =-\int_{0}^{\arcsin \left(\varphi_{t}(\mathbf{a}) \cdot \mathbf{n}(\mathbf{x})\right)} \tan \phi u_{p}^{2}(\sin \phi) d \phi+\frac{(r \Omega \sin \theta)^{2}}{2}+k_{1}, \\
\Phi_{B}(\lambda, \theta, r) & =\frac{(r \Omega \sin \theta)^{2}}{2}+k_{2}
\end{aligned}
$$


are a solution of the SSWE in spherical coordinates. For simplicity, in the remaining terms we choose for $\alpha \in[0, \pi]$ the axis $\mathbf{a}=-\sin \alpha \mathbf{e}_{1}+\cos \alpha \mathbf{e}_{3}$. Thus, we obtain the expressions

$$
\begin{aligned}
\varphi_{t}(\mathbf{a}) \cdot \mathbf{i}= & \sin \alpha(\sin \lambda \cos \Omega t+\cos \lambda \sin \Omega t) \\
\varphi_{t}(\mathbf{a}) \cdot \mathbf{j}= & \sin \alpha \sin \theta(\cos \lambda \cos \Omega t-\sin \lambda \sin \Omega t)+\cos \alpha \cos \theta \\
\varphi_{t}(\mathbf{a}) \cdot \mathbf{n}= & \sin \alpha \cos \theta(-\cos \lambda \cos \Omega t+\sin \lambda \sin \Omega t)+\cos \alpha \sin \theta \\
\varphi_{t}(\mathbf{a}) \times \mathbf{n}= & {[\sin \alpha \sin \theta \sin \Omega t-\cos \alpha \sin \lambda \cos \theta] \mathbf{e}_{1} } \\
& +[\sin \alpha \sin \theta \cos \Omega t+\cos \alpha \cos \lambda \cos \theta] \mathbf{e}_{2} \\
& +[-\sin \alpha \cos \theta(\sin \lambda \cos \Omega t+\cos \lambda \sin \Omega t)] \mathbf{e}_{3} .
\end{aligned}
$$

The orography $\Phi_{B}$ is depicted in fig. 1. Note the unit $\left[1 \mathrm{gpm}=9,81 \frac{\mathrm{m}^{2}}{\mathrm{~s}^{2}}\right]$.

Correspondingly, remark 2 holds for example 2. The solution of example 2 has a time period of 1 day and uses the non-zero orography $\Phi_{B}$. However, it should be stated that the orography $\Phi_{B}$ is not a good approximation of the real Earth's orography.

The following example 3 is a special case of example 2 and describes a solid body rotation with a precession around the Earth's axis, see (Läuter; 2004, 2003).

Example 3 (Unsteady solid body rotation) Let $\mathbf{c} \in \mathbb{R}^{3},|\mathbf{c}|=1$ be a fixed rotation axis, $u_{0} \in \mathbb{R}$ the maximal flow velocity and $k_{1}, k_{2} \in \mathbb{R}$ arbitrary constants. Let $\varphi_{t}$ denote the coordinate transformation function for the rotation in Eq. (11). Then the function $\left(\mathbf{u}_{s b r}, \Phi_{s b r}\right)$, defined for $(\mathbf{x}, t) \in G_{\varepsilon} \times I$

$$
\begin{aligned}
\mathbf{u}_{s b r}(\mathbf{x}, t) & =u_{0} \varphi_{t}(\mathbf{c}) \times \mathbf{n}, \\
\Phi_{s b r}(\mathbf{x}, t) & =\frac{-\left(u_{0} \varphi_{t}(\mathbf{c}) \cdot \mathbf{n}+\boldsymbol{\Omega} \cdot \mathbf{x}\right)^{2}}{2}+\frac{(\boldsymbol{\Omega} \cdot \mathbf{x})^{2}}{2}+k_{1}, \\
\Phi_{B}(\mathbf{x}) & =\frac{(\boldsymbol{\Omega} \cdot \mathbf{x})^{2}}{2}+k_{2}
\end{aligned}
$$

is a solution of the shallow water equations (2) - (4) with the orography $\Phi_{B}$. The derivation consists of an application of example 2. More precisely, we choose the constant $v_{0}=\left|u_{0} \mathbf{c}+a \boldsymbol{\Omega}\right|$, the symmetry axis $\mathbf{a}$ and the velocity profile $u_{p, s b r}$

$$
\mathbf{a}=v_{0}^{-1}\left(u_{0} \mathbf{c}+a \boldsymbol{\Omega}\right), \quad u_{p, s b r}(x)=v_{0} \sqrt{1-x^{2}}, \quad \forall x \in[-1,1],
$$

respectively. With the above values of $\mathbf{a}$ and $u_{p}=u_{p, s b r}$, the fields $\mathbf{u}, \Phi$ and $\Phi_{B}$, defined in example 2, yield the present example. The vorticity and divergence fields of example 2 simplify to

$$
\zeta(\mathbf{x}, t)=\frac{2 u_{0}}{a} \varphi_{t}(\mathbf{c}) \cdot \mathbf{n}, \quad \delta(\mathbf{x}, t)=0
$$

Analog to example 2 this solution may be written in spherical coordinates using the Eqs. (5) - (7). For simplicity, we choose for $\alpha \in[0, \pi]$ the axis $\mathbf{c}=-\sin \alpha \mathbf{e}_{1}+$ 
$\cos \alpha \mathbf{e}_{3}$. We conclude that for all $(\lambda, \theta, r) \in \Sigma_{\varepsilon}, t \in I$ and with $\mathbf{x}=\mathbf{r}(\lambda, \theta, r)$ the functions

$$
\begin{aligned}
u_{s b r}(\lambda, \theta, r, t)= & u_{0}(\sin \alpha \sin \theta(\cos \lambda \cos \Omega t-\sin \lambda \sin \Omega t)+\cos \alpha \cos \theta), \\
v_{s b r}(\lambda, \theta, r, t)= & -u_{0} \sin \alpha(\sin \lambda \cos \Omega t+\cos \lambda \sin \Omega t), \\
\Phi_{s b r}(\lambda, \theta, r, t)=- & \frac{1}{2}\left[u_{0}(\sin \alpha \cos \theta(-\cos \lambda \cos \Omega t+\sin \lambda \sin \Omega t)\right. \\
& +\cos \alpha \sin \theta)+r \Omega \sin \theta]^{2}+\frac{1}{2}(r \Omega \sin \theta)^{2}+k_{1}, \\
\Phi_{B}(\lambda, \theta, r)= & \frac{1}{2}(r \Omega \sin \theta)^{2}+k_{2}
\end{aligned}
$$

are a solution of the SSWE in spherical coordinates. For the example's application in section 5 we have chosen the parameter values $\alpha=\frac{\pi}{4}, u_{0}=\frac{2 \pi a}{12} \frac{\mathrm{m}}{\mathrm{day}}, k_{1}=133681 \frac{\mathrm{m}^{2}}{\mathrm{~s}^{2}}$ and $k_{2}=0 \frac{\mathrm{m}^{2}}{\mathrm{~s}^{2}}$. The velocity $\mathbf{u}_{\text {sbr }}$ and the geopotential $\Phi_{\text {sbr }}$ at the initial time $t=0 \mathrm{~s}$ are depicted in fig. 2.

Correspondingly, remark 2 holds for example 3. For $\alpha=0$ example 3 corresponds to Williamson et al. (1992, case 2), but with a different orography. With the presented transformation method it is not obvious to give an unsteady example of an axially symmetric flow with compact support, as described in the steady state case e. g. in Galewsky et al. (2004) and Browning et al. (1989). However, the following special case of example 2 describes an unsteady solid body rotation with a superimposed tropospheric jet.

Example 4 (Unsteady jet stream) Let $\mathbf{c} \in \mathbb{R}^{3},|\mathbf{c}|=1$ be a fixed rotation axis, $u_{0}, u_{1} \in \mathbb{R}$ flow velocities, $\theta_{0}, \theta_{1} \in\left(-\frac{\pi}{2}, \frac{\pi}{2}\right)$ latitudinal angles and $k_{1}, k_{2} \in \mathbb{R}$ arbitrary constants. Let $\varphi_{t}$ denote the coordinate transformation function for the rotation in Eq. (11). Further on let $\mathbf{u}_{s b r}$ and $\Phi_{s b r}$ be the fields in Eq. (23) and let $\mathbf{a}$ and $u_{p, s b r}$ be defined by $E q$. (24). With $c_{0}=u_{1} \exp \left(\frac{4}{\left(\theta_{0}-\theta_{1}\right)^{2}}\right)$, we define the velocity profile of the jet stream for all $x \in[-1,1]$ by

$$
u_{p, j e t}(x)=\left\{\begin{array}{ll}
c_{0} \exp \left(\frac{1}{\left(\arcsin x-\theta_{0}\right)\left(\arcsin x-\theta_{1}\right)}\right) & \text { for } \theta_{0} \leq \arcsin x \leq \theta_{1} \\
0 & \text { else }
\end{array} .\right.
$$

Then the function $(\mathbf{u}, \Phi)$, defined for all $(\mathbf{x}, t) \in G_{\varepsilon} \times I$

$$
\begin{aligned}
& \mathbf{u}(\mathbf{x}, t)=\mathbf{u}_{s b r}(\mathbf{x}, t)+u_{p, j e t}\left(\varphi_{t}(\mathbf{a}) \cdot \mathbf{n}\right) \frac{\varphi_{t}(\mathbf{a}) \times \mathbf{n}}{\left|\varphi_{t}(\mathbf{a}) \times \mathbf{n}\right|}, \\
& \Phi(\mathbf{x}, t)=\Phi_{s b r}(\mathbf{x}, t)-\int_{0}^{\arcsin \left(\varphi_{t}(\mathbf{a}) \cdot \mathbf{n}\right)} \tan \phi\left(2 u_{p, s b r} u_{p, j e t}+u_{p, j e t}^{2}\right) \circ \sin \phi d \phi+k_{1}, \\
& \Phi_{B}(\mathbf{x})=\frac{(\boldsymbol{\Omega} \cdot \mathbf{x})^{2}}{2}+k_{2}
\end{aligned}
$$


is a solution of SSWE (2) - (4) with the orography $\Phi_{B}$. The derivation is similar to example 3. More precisely, it is an application of example 2 with the symmetry axis $\mathbf{a}$ and the velocity profile $u_{p}=u_{p, s b r}+u_{p, j e t}$. The representation of vorticity $\zeta$ and divergence $\delta$ is the same as in example 2, but can not be simplified here.

Analog to example 2 this solution may be written in spherical coordinates using the Eqs. (5) - (7). For simplicity, we choose for $\alpha \in[0, \pi]$ the axis $\mathbf{c}=-\sin \alpha \mathbf{e}_{1}+$ $\cos \alpha \mathbf{e}_{3}$ and after the axis a by Eq. (24). For all $(\lambda, \theta, r) \in \Sigma_{\varepsilon}, t \in I$ and with $\mathbf{x}=\mathbf{r}(\lambda, \theta, r)$ we obtain, using Eq. (22), the functions

$$
\begin{aligned}
u(\lambda, \theta, r, t)= & u_{s b r}(\lambda, \theta, r, t)+\frac{u_{p, j e t}\left(\varphi_{t}(\mathbf{a}) \cdot \mathbf{n}\right)}{\left|\varphi_{t}(\mathbf{a}) \times \mathbf{n}\right|} \varphi_{t}(\mathbf{a}) \cdot \mathbf{j}, \\
v(\lambda, \theta, r, t)= & v_{s b r}(\lambda, \theta, r, t)-\frac{u_{p, j e t}\left(\varphi_{t}(\mathbf{a}) \cdot \mathbf{n}\right)}{\left|\varphi_{t}(\mathbf{a}) \times \mathbf{n}\right|} \varphi_{t}(\mathbf{a}) \cdot \mathbf{i}, \\
\Phi(\lambda, \theta, r, t)= & \Phi_{s b r}(\lambda, \theta, r, t) \\
& -\int_{0}^{\arcsin \left(\varphi_{t}(\mathbf{a}) \cdot \mathbf{n}\right)} \tan \phi\left(2 u_{p, s b r} u_{p, j e t}+u_{p, j e t}^{2}\right) \circ \sin \phi d \phi+k_{1}, \\
\Phi_{B}(\lambda, \theta, r)= & \frac{1}{2}(r \Omega \sin \theta)^{2}+k_{2}
\end{aligned}
$$

which are a solution of the SSWE in spherical coordinates. Here, $u_{s b r}, v_{s b r}$ and $\Phi_{s b r}$ denote the solution in spherical coordinates of example 3. For the example's application in section 5 we have chosen the parameter values $\alpha=\frac{\pi}{4}, u_{0}=20 \frac{\mathrm{m}}{\mathrm{s}}$, $u_{1}=\frac{2 \pi a}{12} \frac{\mathrm{m}}{\mathrm{day}}, \theta_{0}=\frac{\pi}{7}, \theta_{1}=\frac{\pi}{2}-\theta_{0}, k_{1}=130905 \frac{\mathrm{m}^{2}}{\mathrm{~s}^{2}}$ and $k_{2}=0 \frac{\mathrm{m}^{2}}{\mathrm{~s}^{2}}$. The velocity $\mathbf{u}$ and the geopotential $\Phi$ at the initial time $t=0$ s are depicted in fig. 3.

Correspondingly, remark 2 holds for example 4.

\section{Convergence properties of the atmospheric model PLASMA}

In this section we quantify the convergence properties of the atmospheric model PLASMA (Parallel Large Scale Model of the Atmosphere) by means of the steady state solution (Williamson et al.; 1992, case 2) and the unsteady analytical solutions from section 4.2. Several features of the model PLASMA have been published recently, concerning the numerical method in Läuter (2003, 2004), concerning the grid generation in Behrens et al. (2005) and concerning the matrix solver in Frickenhaus et al. (2005). The reader may contact the authors by e-mail to get a FORTRAN 90 code for the unsteady solutions in section 4.2 .

The model equations of PLASMA are the SSWE (2) - (4) in scalar formulation with 
the prognostic variables vorticity $\zeta$, divergence $\delta$ and geopotential $\Phi$.

$$
\begin{aligned}
\partial_{t} \zeta+\mathbf{u} \cdot \nabla_{S} \zeta+\zeta \delta+f \delta & =-\mathbf{u} \cdot \nabla_{S} f \\
\partial_{t} \delta+\mathbf{u} \cdot \nabla_{S} \delta+\Delta_{S} \Phi-f \zeta & =-(\mathbf{n} \times \mathbf{u}) \cdot \nabla_{S} f \\
& \quad-\sum_{i, j=1}^{3}\left(\nabla_{S} u_{i}\right)_{j}\left(\nabla_{S} u_{j}\right)_{i}-\mathbf{u} \cdot \mathbf{u} \\
\partial_{t} \Phi+\mathbf{u} \cdot \nabla_{S}\left(\Phi-\Phi_{B}\right)+\left(\Phi-\Phi_{B}\right) \delta & =0 \\
-\Delta_{S} \psi & =\zeta, \\
\Delta_{S} \chi & =\delta, \\
\operatorname{rot}_{S} \psi+\nabla_{S} \chi & =\mathbf{u}
\end{aligned}
$$

For the numerical implementation of the SSWE the Lagrange-Galerkin method has been applied. This method combines the semi-Lagrangian method with the finite element method. The semi-Lagrangian method, which has good stability properties, discretizes the material derivative along trajectories. The finite element method on the sphere is constructed on an approximating polyhedron consisting of a triangular grid with nodes on the sphere.

To quantify the model's convergence properties we choose the relative $L^{2}$-error

$$
\eta(g)=\frac{\left\|g-g_{a n a}\right\|_{S}}{\left\|g_{\text {ana }}\right\|_{S}}
$$

for a numerical solution $g: S \rightarrow \mathbb{R}$, its according analytical solution $g_{\text {ana }}: S \rightarrow \mathbb{R}$ and the $L^{2}$-norm $\|g\|_{S}=\left(\int_{S}|g|^{2} d \sigma\right)^{\frac{1}{2}}$. In the following two sections we regard the $L^{2}$-error of the vorticity $\zeta$ and the geopotential $\Phi$ after a simulation time of $12 \mathrm{~h}$. Due to the fact that in all cases the divergence $\delta_{a n a}$ vanishes a relative error of $\delta$ is not computable. Because the convergence analysis for $\delta$ with the absolute $L^{2}$-error $\|\delta\|_{S}$ qualitatively shows the same results as for $\zeta$, see (Läuter; 2004), we confine our error analysis to $\zeta$ and $\Phi$.

\subsection{A steady state solution}

At first, we consider the solid body rotation given in Williamson et al. (1992, case 2). The relative errors $\eta(\zeta)$ and $\eta(\Phi)$ after a simulation time of $12 \mathrm{~h}$ are depicted in fig. 4 in dependence on the time step $\Delta t$ and the grid resolution $\Delta x$. While the errors are almost independent of $\Delta t$, the errors decrease monotonously as $\Delta x$ decreases until the minima $\eta(\zeta)=2.5 \cdot 10^{-4}$ and $\eta(\Phi)=4.0 \cdot 10^{-5}$. This order of magnitude seems to be reasonable due to the model's single precision accuracy. Contrary to the expectation, for $\Delta t=2 \mathrm{~min}$ the monotony of $\eta$ fails at $\Delta x=261 \mathrm{~km}$. Probably, here the computational truncation dominates the discretization error. This will be a matter of future analysis with double precision accuracy. Fig. 5 shows the spatial error distribution of $\Phi-\Phi_{a n a}$. $\Phi-\Phi_{\text {ana }}$ is limited by $4 \mathrm{gpm}$ and the error structure displays a zonal wave number five which is an effect of the icosahedral grid structure. 


\section{$5.2 \quad$ Unsteady solutions}

The simulation of the unsteady solid body rotation of example 3 allows us to study the convergence properties for unsteady flows. $\eta(\zeta)$ and $\eta(\Phi)$ are depicted in fig. 6 after a simulation time of $12 \mathrm{~h}$. In contrast to the steady state solution in section 5.1 the errors are almost independent of $\Delta x$, but decrease as $\Delta t$ decreases. This seems to be a feature of the semi-Lagrangian method. During a time step from $t_{0}$ to $t_{0}+\Delta t$ the flow trajectory depends on the velocity field $\mathbf{u}$, which is a function of space and time $t \in\left[t_{0}, t_{0}+\Delta t\right]$. Because $\mathbf{u}$ is a model variable, the discrete trajectory has to be evaluated only for $\mathbf{u}$-values for $t \leq t_{0}$. For the steady state solution in section $5.1 \mathbf{u}$ is independent on time. Thus, the discrete trajectory is independent on $\Delta t$ for sufficient small $\Delta t$. In contrast, for the unsteady solution the $\mathbf{u}$-values for $t \in\left[t_{0}, t_{0}+\Delta t\right]$ are extrapolated by the values for $t \leq t_{0}$. Thus, the calculation of the discrete trajectory improves for decreasing $\Delta t$. Due to the smooth fields in this case the trajectory error dominates $\eta$. The resulting minima $\eta(\zeta)=2.0 \cdot 10^{-2}$ and $\eta(\Phi)=5.0 \cdot 10^{-4}$ are 2 respectively 1 order of magnitude larger compared to the results of the steady state solution. Fig. 7 shows the spatial error distribution of $\Phi-\Phi_{a n a}$. The maximum of about $40 \mathrm{gpm}$ is noticeable higher than in the steady state case, but the error does not reveal any zonal wave number five structure. Compared to fig. 2, we find an error distribution symmetric to the solid body rotation axis, instead (notice that fig. 2 shows the geopotential at initial time $t=0)$. Finally, small-scale gravity wave structures appear near the points $\left(-100^{\circ}, 40^{\circ}\right)$ and $\left(80^{\circ},-40^{\circ}\right)$.

Example 4, the unsteady solid body rotation with superimposed jet stream, is chosen as a second unsteady test case. $\eta(\zeta)$ and $\eta(\Phi)$ are depicted in fig. 8 after a simulation time of $12 \mathrm{~h}$. Although there are slight dependencies on $\Delta t, \eta(\zeta)$ and $\eta(\Phi)$ primarily depend on $\Delta x$. This is due to the sharp gradients near the jet stream which were not present in example 3. Thus, now the spatial discretiztion error dominates the trajectory error. The significant oscillation for $\eta(\Phi)$ we attribute to the grid structure. The grid triangles are generated by repeated bisection. For two consecutive grids with, e.g. $\Delta x=522 \mathrm{~km}$ and $\Delta x=428 \mathrm{~km}$, sections of the grids are depicted in the figures 10 and 11, respectively. In fig. 10 we find more edges orientated parallelly to the flow direction than in fig. 11. This orientation causes smoother discrete fields for $\Delta x=522 \mathrm{~km}$ than for $\Delta x=428 \mathrm{~km}$ although the grid resolution is coarser. The resulting minima of $\eta(\zeta)=4.0 \cdot 10^{-2}$ and $\eta(\Phi)=9.0 \cdot 10^{-4}$ are similar to the values for the unsteady solid body rotation. Fig. 9 shows the spatial error distribution of $\Phi-\Phi_{\text {ana }}$. Again, the maximum value of about $250 \mathrm{gpm}$ is one order of magnitude larger than for the unsteady solid body rotation. Compared to fig. 3, we find the error maxima near strong geopotential gradients (notice that fig. 3 shows the geopotential at initial time $t=0$ ). Additionally, all contour lines show small-scale gravity wave structures.

The presented application of unsteady analytical solutions for the convergence analysis of the model PLASMA provides information for a further model development. For realistic flow, which is unsteady in particular, an unsteady analytical solution test gives a better estimation of the numerical error than a steady state solution 
test. The errors for the unsteady tests are about 2 orders of magnitude larger than for the steady state test. The error dependence on the time step in fig. 6 shows that the choice of time step in the model is not only a question of model stability but of accuracy of the model result.

\section{Summary}

Models based on the SSWE are one way to realize a barotropic model of the atmosphere. For the validation process the quantification of convergence properties is an important task. Therefore, the necessary objective error measures are provided by analytical solutions of the SSWE. Whereas steady state analytical solutions which consist essentially in axially symmetric solutions, are well known from the literature, unsteady analytical solutions of the SSWE have not been established until now.

We presented the transformation method for the SSWE which is based on the change from a fixed cartesian to a rotating coordinate system. The fundamental examples in section 4 provide unsteady analytical solutions of the SSWE. They consist of axially symmetric flows and include a solid body rotation and a jet stream as special cases.

Based on the given unsteady solutions we have shown a quantification of the convergence properties of the atmospheric model PLASMA. The extension of the analysis from steady state to unsteady solutions demonstrates new demands on model accuracy in space and time. The requirements for the model time step are on the one hand model stability and on the other hand the model accuracy. Thus the application of the unsteady analytical solutions to the model validation gives important hints on further model development.

\section{Acknowledgements}

This work is supported by the joint project PLASMA (Parallel LArge Scale Model of the Atmosphere), which is founded in the framework of DEKLIM (German climate research project) by the Federal Ministry of Education and Research of Germany (grant number $01 \mathrm{LD}$ 0037). The authors thank the two anonymous reviewers for useful hints which improved the manuscript.

\section{A General curvilinear coordinates}

The concept of general curvilinear coordinates is a method to describe functions with respect to different coordinates. We distinguish between the generic coordinates and the curvilinear coordinates. The generic coordinates are the coordinates with respect to the generic basis vectors

$$
\mathbf{e}_{i}=(1,0,0)^{\mathrm{T}}, \quad \mathbf{e}_{i}=(0,1,0)^{\mathrm{T}}, \quad \mathbf{e}_{i}=(0,0,1)^{\mathrm{T}}
$$


in $\mathbb{R}^{3}$. The curvilinear coordinates are defined by their coordinate transformation function $\varphi_{t}$, which defines the mapping from the generic to the curvilinear coordinates. For open sets $G, \Sigma \subset \mathbb{R}^{3}$ we denote the coordinate transformation function for $t \in I$ with $\varphi_{t}: G \rightarrow \Sigma$ and its inverse with $\varphi_{t}^{-1}: \Sigma \rightarrow G$. Then for a generic coordinate $\mathbf{x} \in G$ and the corresponding curvilinear coordinate $\mathbf{y} \in \Sigma$ the equations

$$
\varphi_{t}(\mathbf{x})=\mathbf{y}, \quad \varphi_{t}^{-1}(\mathbf{y})=\mathbf{x}
$$

hold. Further on the position vector $\mathbf{r}: \Sigma \rightarrow G$ of a curvilinear coordinate $\mathbf{y} \in \Sigma$ is defined by $\mathbf{r}(\mathbf{y}, t)=\varphi_{t}^{-1}(\mathbf{y})$. We define the velocity $\mathbf{w}$ of a particle in the generic coordinate system which is resting in the curvilinear coordinates by

$$
\mathbf{w}(\mathbf{x}, t)=\frac{\partial \mathbf{r}}{\partial t}\left(\varphi_{t}(\mathbf{x}), t\right), \quad \forall(\mathbf{x}, t) \in G \times I .
$$

The description of velocities in general curvilinear coordinates is based on the movement of a Lagrangian particle along a trajectory. A particle's trajectory can be described by trajectory functions $\mathbf{x}: I \rightarrow G$ and $\mathbf{y}: I \rightarrow \Sigma$ in generic and curvilinear coordinates, respectively. By definition, these functions fulfill the equation $\mathbf{x}(t)=\mathbf{r}(\mathbf{y}(t), t)$ for all $t \in I$. Then the particle's velocities $\mathbf{v}: I \rightarrow \mathbb{R}^{3}$ and $\mathbf{u}: I \rightarrow \mathbb{R}^{3}$ in generic and curvilinear coordinates, respectively, are defined by $\mathbf{v}(t)=\frac{\partial \mathbf{x}}{\partial t}$ and $\mathbf{u}(t)=\frac{\partial \mathbf{y}}{\partial t}=\frac{\partial y_{i}}{\partial t} \mathbf{e}_{i}$. For all $t \in I$, one obtains the relation between $\mathbf{v}$ and $\mathbf{u}$ by applying Eq. (26)

$$
\mathbf{v}(t)=\frac{d \mathbf{r}(\mathbf{y}(t), t)}{d t}=\mathbf{w}(\mathbf{r}(\mathbf{y}, t), t)+\mathbf{u}(t) \cdot \mathbf{e}_{i} \frac{\partial \mathbf{r}}{\partial y_{i}}(\mathbf{y}, t)
$$

Due to this relation, the two velocity fields $\mathbf{v}: G \rightarrow \mathbb{R}^{3}$ and $\mathbf{u}: \Sigma \rightarrow \mathbb{R}^{3}$ in generic and curvilinear coordinates, respectively, describe the same physical flow, if they fulfill the equation

$$
\mathbf{v}=\mathbf{u} \cdot \mathbf{e}_{i} \frac{\partial \mathbf{r}}{\partial y_{i}}+\mathbf{w}
$$

\section{References}

Behrens, J., Rakowsky, N., Hiller, W., Handorf, D., Läuter, M., Päpke, J. and Dethloff, K. (2005). amatos: parallel adaptive mesh generator for atmospheric and oceanic simulation, Ocean Modelling 10: 171-183.

Bonaventura, L. and Ringler, T. R. (2004). Analysis of discrete shallow water models on geodesic Delauny grids with C-type staggering, Mon. Wea. Rev. ., submitted.

Browning, G. L., Hack, J. J. and Swarztrauber, P. N. (1989). A comparison of three numerical methods for solving differential equations on the sphere, Mon. Wea. Rev. 117: 1058-1075.

Côté, J. (1988). A Lagrange multiplier approach for the metric terms of semiLagrangian models on the sphere, Q. J. R. Meteorol. Soc. 114: 1347-1352. 
Preprint 2005, Alfred Wegener Institute, Germany, M. Läuter, D. Handorf, K. Dethloff

Côté, J. and Staniforth, A. (1990). An accurate and efficient finite-element global model of the shallow-water equations, Mon. Wea. Rev. 118: 2707-2717.

Dee, D. P. and Da Silva, A. M. (1986). Using Hough harmonics to validate and assess nonlinear shallow-water models, Mon. Wea. Rev. 114: 2191-2196.

Dey, C. H. (1969). A note on global forecasting with the Kurihara grid, Mon. Wea. Rev. 97: 597-601.

Dutton, J. A. (1995). Dynamics of atmospheric motion, Dover Publications, New York.

Frickenhaus, S., Hiller, W. and Best, M. (2005). FoSSI: Family of simplified solver interfaces for parallel sparse solvers in numerical atmosphere and ocean modeling, Ocean Modelling 10: 185-191.

Galewsky, J., Scott, R. and Polvani, L. M. (2004). An initial-value problem for testing numerical models of the global shallow water equations, Tellus A 56: 429440 .

Giraldo, F. X. (2001). A spectral element shallow water model on spherical geodesic grids, Int. J. Numer. Meth. Fluids 35: 869-901.

Giraldo, F. X. and Warburton, T. (2005). A nodal triangle-based spectral element method for the spherical shallow water equations on unstructured grids, J. Comput. Phys. 207: 129-150.

Haltiner, G. J. and Williams, R. (1980). Numerical Prediction and Dynamic Meteorology, Wiley, New York.

Heinze, T. and Hense, A. (2002). The shallow water equations on the sphere and their Lagrange-Galerkin solution, Meteorol. Atmos. Phys. 81: 129-137.

Hoskins, B. J. (1973). Stability of the Rossby-Haurwitz wave, Q. J. R. Meteorol. Soc. 99: 723-745.

Jablonowski, C. (2004). Adaptive grids in weather and climate modeling, PhD thesis, The University of Michigan, USA.

Jakob-Chien, R., Hack, J. J. and Williamson, D. L. (1995). Spectral transform solutions to the shallow water test set, J. Comput. Phys. 119: 164-187.

Läuter, M. (2003). An adaptive Lagrange-Galerkin method for the shallow-water equations on the sphere, PAMM - Proceedings in Applied Mathematics and Mechanics 3: 48-51.

Läuter, M. (2004). Großräumige Zirkulationsstrukturen in einem nichtlinearen adaptiven Atmosphärenmodell, PhD thesis, Universität Potsdam, Am Neuen Palais 10, 14469 Potsdam, Germany. 
Lin, S.-J. and Rood, R. B. (1997). An explicit flux-form semi-Lagrangian shallowwater model on the sphere, Q. J. R. Meteorol. Soc. 123: 2477-2498.

McDonald, A. and Bates, J. R. (1989). Semi-Lagrangian integration of a gridpoint shallow water model on the sphere, Mon. Wea. Rev. 117: 130-137.

Merilees, P. E., Ducharme, P. and Jacques, G. (1977). Experiments with a polar filter and a one-dimensional semi-implicit algorithm, Atmosphere 15: 19-33.

Pedlosky, J. (1987). Geophysical Fluid Dynamics, Springer, New York.

Phillips, N. A. (1959). Numerical integration of the primitive equations on the hemisphere, Mon. Wea. Rev. 87: 333-345.

Piani, C. and Norton, W. A. (2002). Solid-body rotation in the northern hemisphere summer stratosphere, Geophys. Res. Lett. 29: 2117-2120.

Ringler, T. D. and Randall, D. A. (2002). A potential enstrophy and energy conserving numerical scheme for solution of the shallow-water equations on a geodesic grid, Mon. Wea. Rev. 130(5): 1397-1410.

Stuhne, G. R. and Peltier, W. R. (1999). New icosahedral grid-point discretizations of the shallow water equations on the sphere, J. Comput. Phys. 148: 23-58.

Swarztrauber, P. N., Williamson, D. L. and Drake, J. B. (1997). The cartesian method for solving partial differential equations in spherical geometry, Dyn. Atmos. Oceans 27: 679-706.

Takacs, L. L. (1988). Effects of using a posteriori methods for the conservation of integral invariants, Mon. Wea. Rev. 116: 525-545.

Tolstykh, M. A. (2002). Vorticity-divergence semi-Lagrangian shallow-water model of the sphere based on compact finite differences, J. Comput. Phys. 179: 180-200.

Tomita, H., Tsugawa, M., Satoh, M. and Goto, K. (2001). Shallow water model on a modified icosahedral geodesic grid by using spring dynamics, J. Comput. Phys. 174: 579-613.

Umscheid, L. and Sankar-Rao, M. (1971). Further tests of a grid system for global numerical prediction, Mon. Wea. Rev. 99: 686-690.

Williamson, D. L. and Browning, G. L. (1973). Comparison of grids and difference approximations for numerical weather prediction over a sphere, J. Appl. Meteorol. 12: $264-274$.

Williamson, D. L., Drake, J. B., Hack, J. J., Jakob, R. and Swarztrauber, P. N. (1992). A standard test set for numerical approximations to the shallow water equations in spherical geometry, J. Comput. Phys. 102: 211-224. 


\section{List of Figures}

1 Orography $\Phi_{B}$, example $2 \ldots \ldots \ldots$. . . . . . . . . . . 22

2 Velocity $\mathbf{u}_{s b r}$, geopotential $\Phi_{s b r}$, example $3 \ldots \ldots . . \ldots 23$

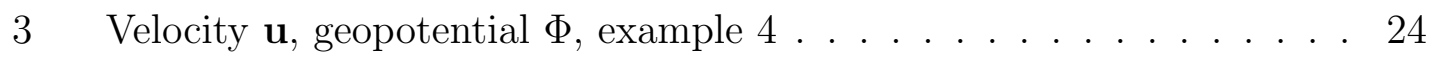

4 Steady state solid body rotation, rel. errors for vorticity $\eta(\zeta)$ and geopotential $\eta(\Phi)$, time step $\Delta t$, grid resolution $\Delta x \ldots 25$

5 Steady state solid body rotation, difference plot $\Phi-\Phi_{\text {ana }} \ldots \ldots$. . . 26

6 Unsteady solid body rotation, rel. errors for vorticity $\eta(\zeta)$ and geopotential $\eta(\Phi)$, time step $\Delta t$, grid resolution $\Delta x \ldots \ldots . \ldots . \ldots 27$

$7 \quad$ Unsteady solid body rotation, difference plot $\Phi-\Phi_{\text {ana }} \quad \ldots \ldots$. . . . 28

8 Unsteady jet stream, rel. errors for vorticity $\eta(\zeta)$ and geopotential

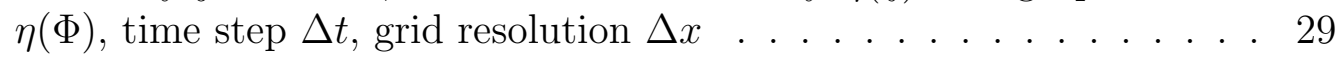

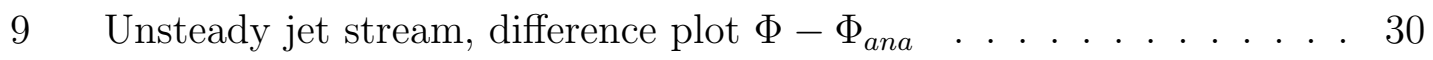

10 Unsteady jet stream, velocity $\mathbf{u}$, geopotential $\Phi, \Delta x=522 \mathrm{~km}$, section $\left(-10^{\circ}, 10^{\circ}\right) \times\left(35^{\circ}, 45^{\circ}\right) \ldots \ldots \ldots . \ldots \ldots$

11 Unsteady jet stream, velocity $\mathbf{u}$, geopotential $\Phi, \Delta x=428 \mathrm{~km}$, sec-

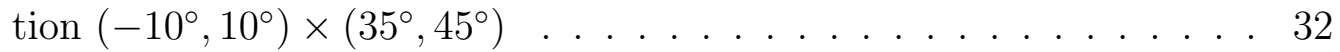


Preprint 2005, Alfred Wegener Institute, Germany, M. Läuter, D. Handorf, K. Dethloff

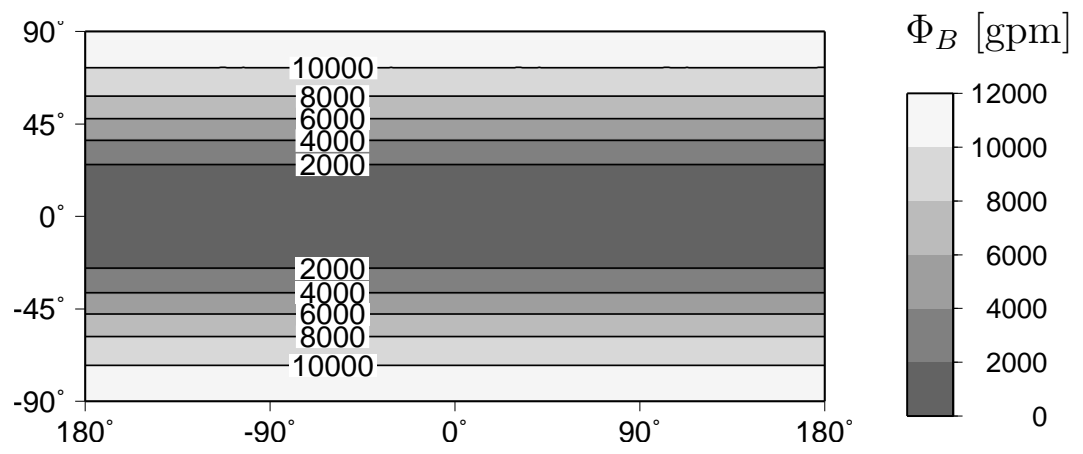

Figure 1: Orography $\Phi_{B}$, example 2 
Preprint 2005, Alfred Wegener Institute, Germany, M. Läuter, D. Handorf, K. Dethloff

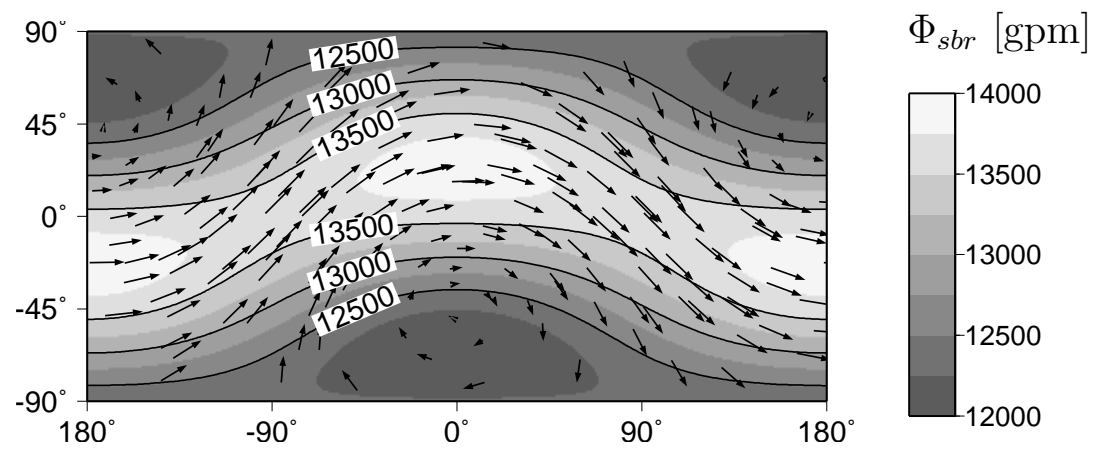

Figure 2: Velocity $\mathbf{u}_{s b r}$, geopotential $\Phi_{s b r}$, example 3 
Preprint 2005, Alfred Wegener Institute, Germany, M. Läuter, D. Handorf, K. Dethloff

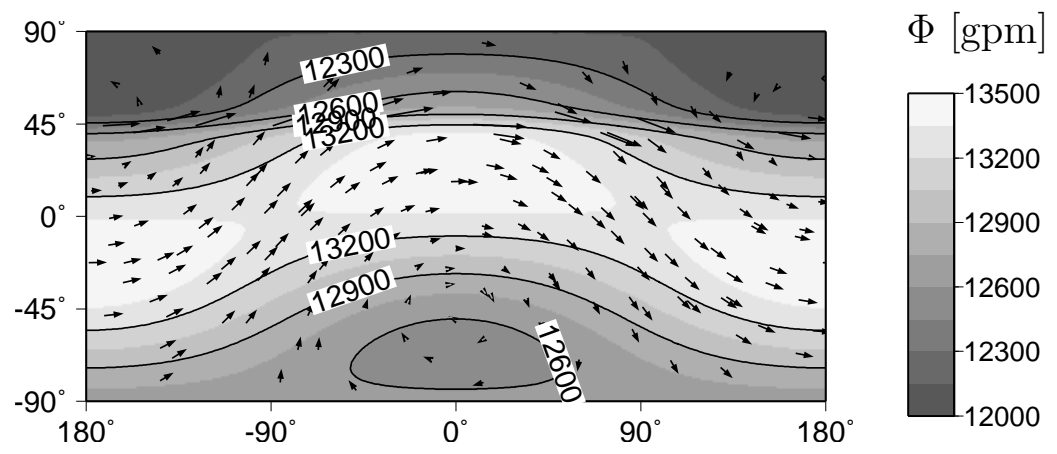

Figure 3: Velocity $\mathbf{u}$, geopotential $\Phi$, example 4 
Preprint 2005, Alfred Wegener Institute, Germany, M. Läuter, D. Handorf, K. Dethloff
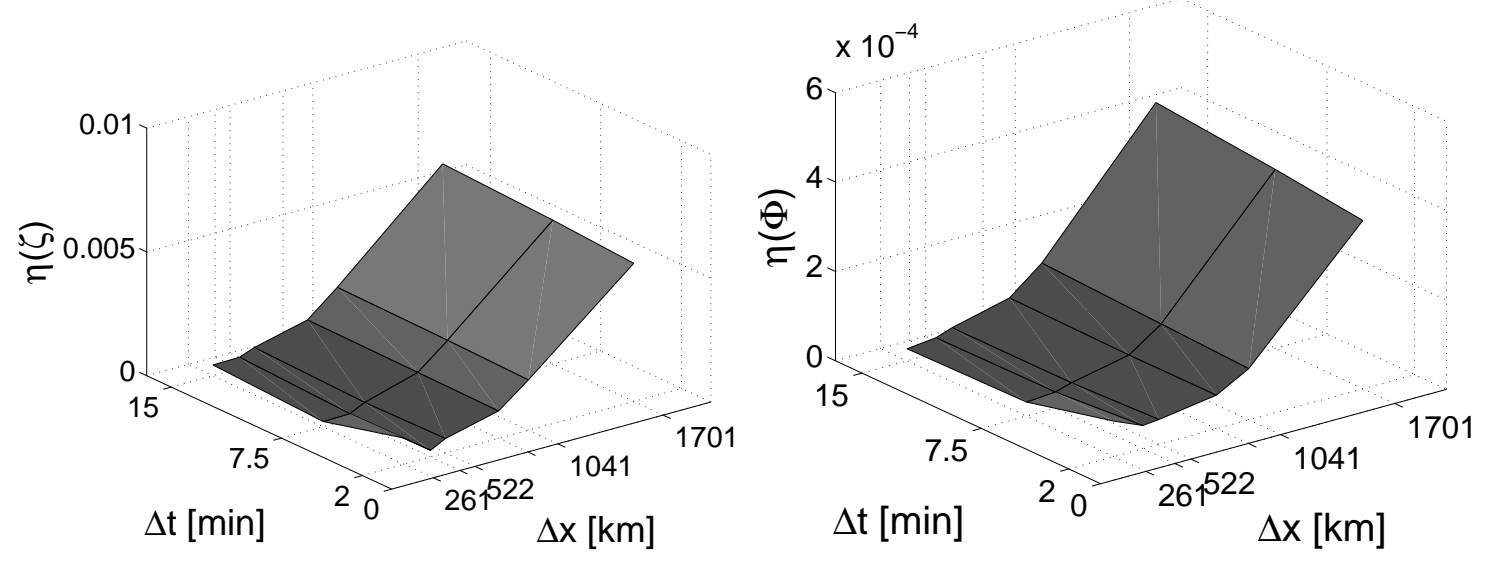

Figure 4: Steady state solid body rotation, rel. errors for vorticity $\eta(\zeta)$ and geopotential $\eta(\Phi)$, time step $\Delta t$, grid resolution $\Delta x$ 
Preprint 2005, Alfred Wegener Institute, Germany, M. Läuter, D. Handorf, K. Dethloff

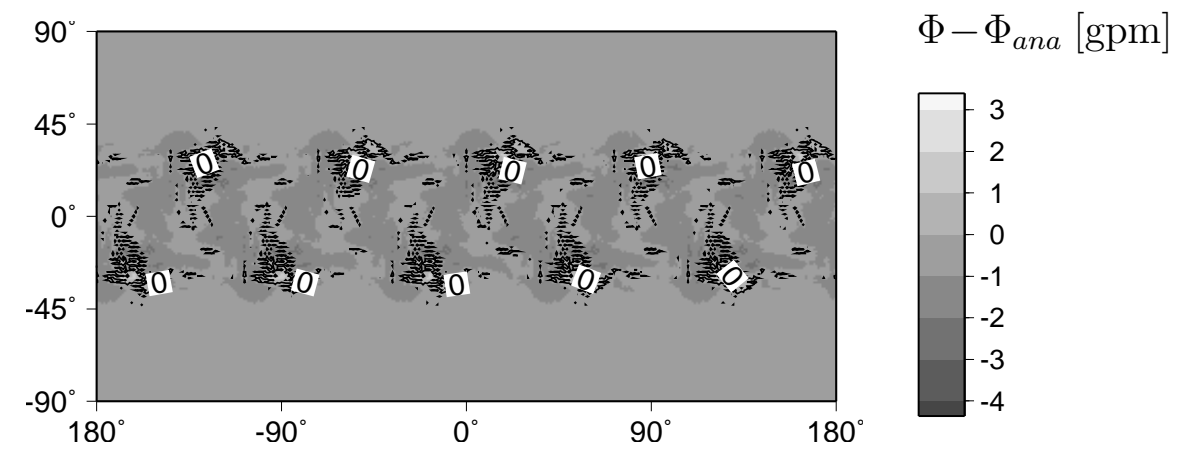

Figure 5: Steady state solid body rotation, difference plot $\Phi-\Phi_{\text {ana }}$ 
Preprint 2005, Alfred Wegener Institute, Germany, M. Läuter, D. Handorf, K. Dethloff
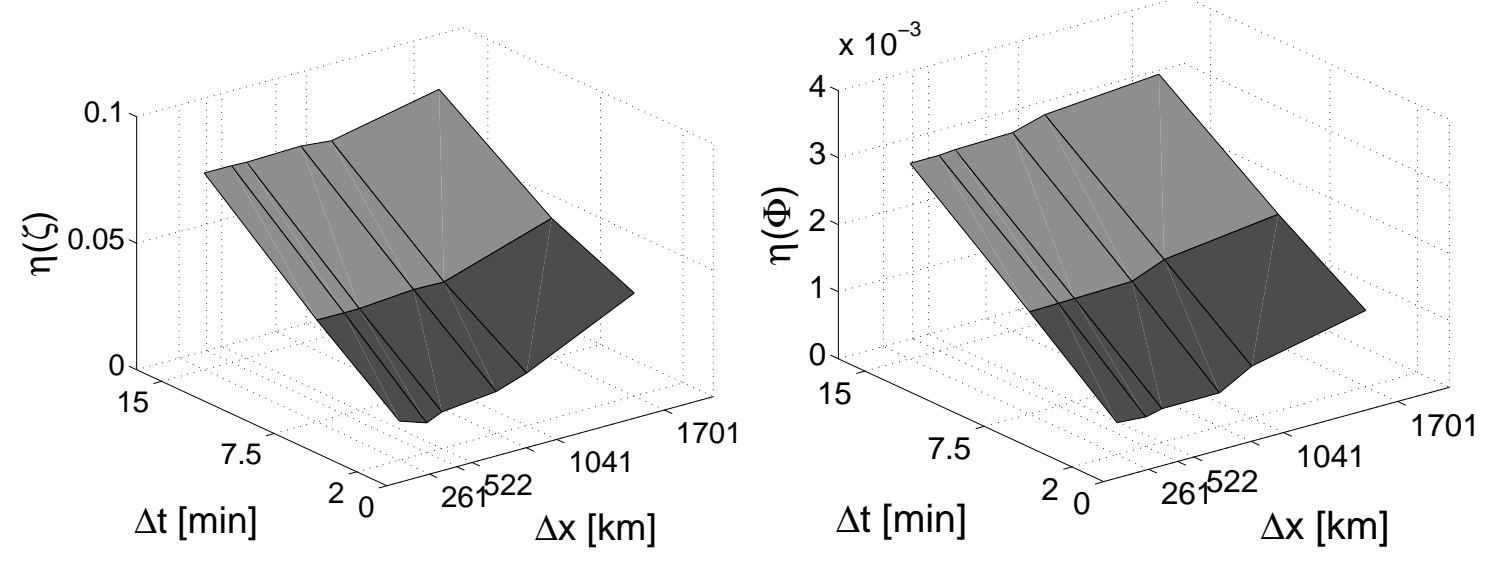

Figure 6: Unsteady solid body rotation, rel. errors for vorticity $\eta(\zeta)$ and geopotential $\eta(\Phi)$, time step $\Delta t$, grid resolution $\Delta x$ 
Preprint 2005, Alfred Wegener Institute, Germany, M. Läuter, D. Handorf, K. Dethloff

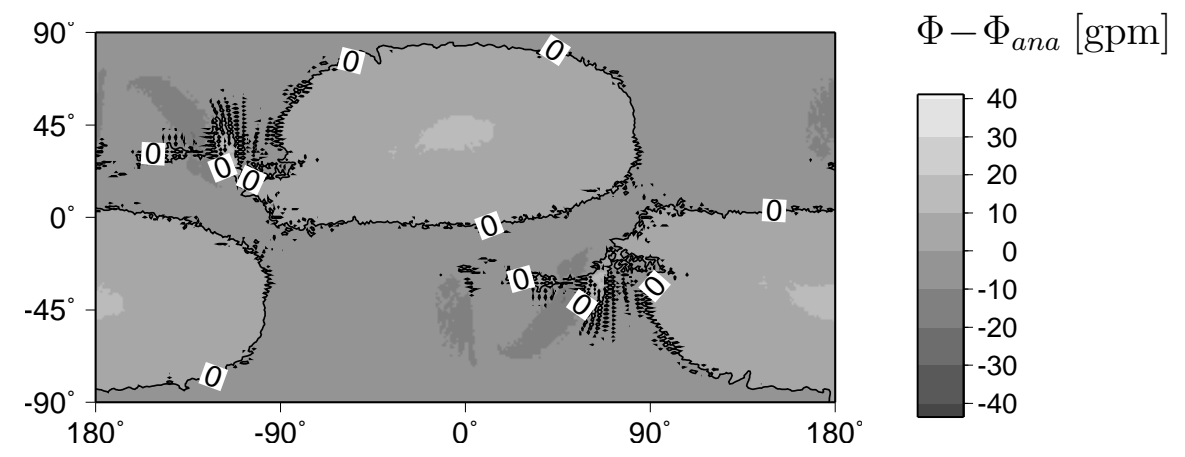

Figure 7: Unsteady solid body rotation, difference plot $\Phi-\Phi_{a n a}$ 
Preprint 2005, Alfred Wegener Institute, Germany, M. Läuter, D. Handorf, K. Dethloff
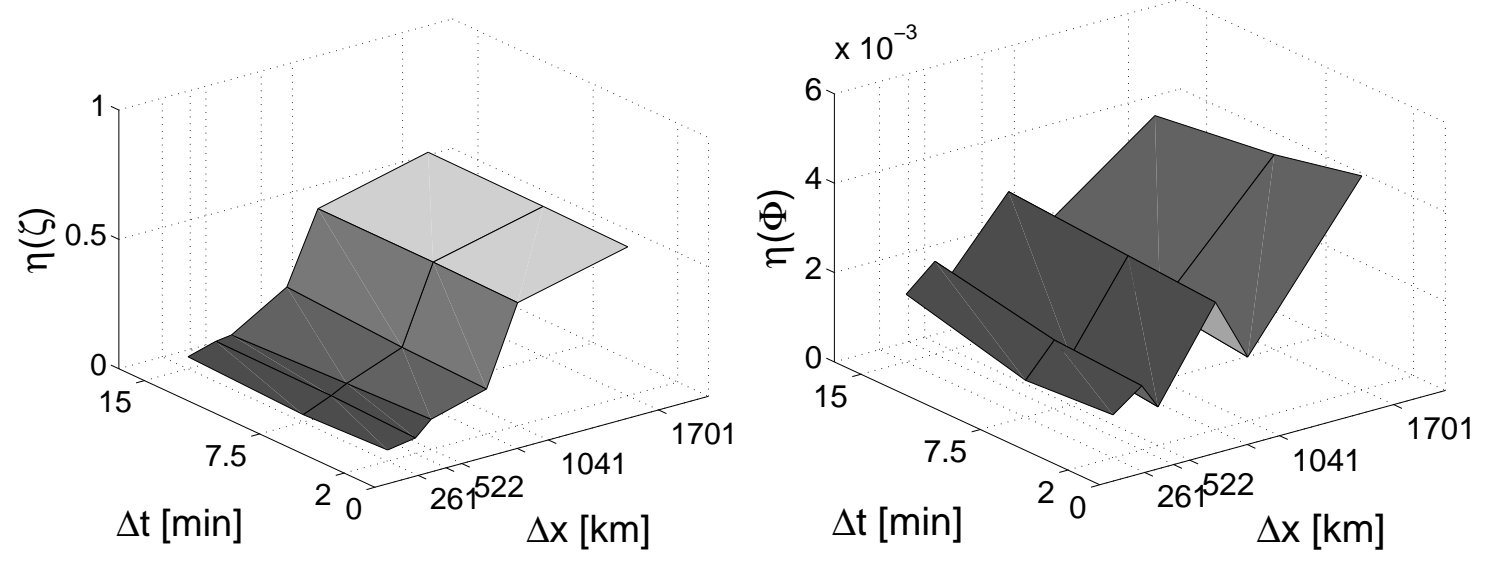

Figure 8: Unsteady jet stream, rel. errors for vorticity $\eta(\zeta)$ and geopotential $\eta(\Phi)$, time step $\Delta t$, grid resolution $\Delta x$ 
Preprint 2005, Alfred Wegener Institute, Germany, M. Läuter, D. Handorf, K. Dethloff

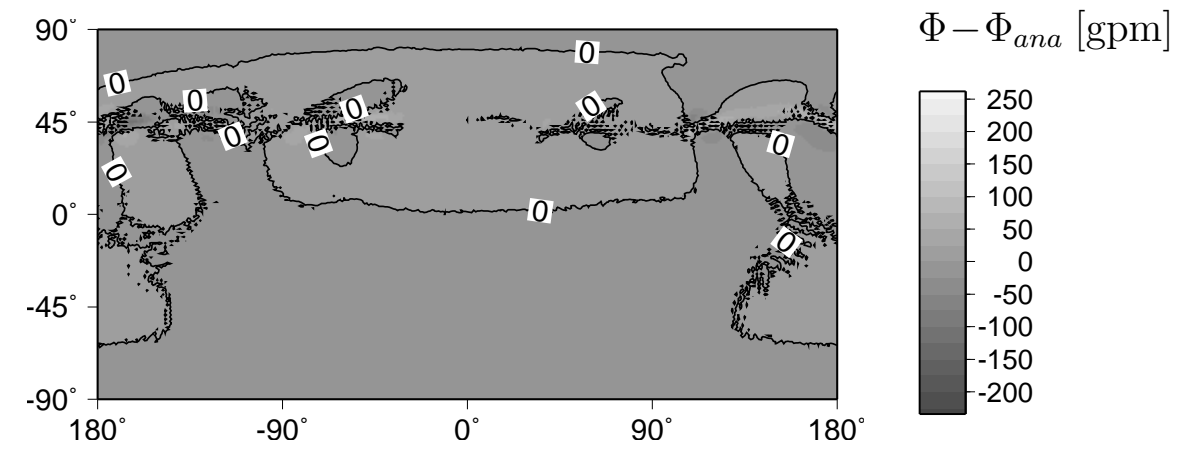

Figure 9: Unsteady jet stream, difference plot $\Phi-\Phi_{\text {ana }}$ 
Preprint 2005, Alfred Wegener Institute, Germany, M. Läuter, D. Handorf, K. Dethloff

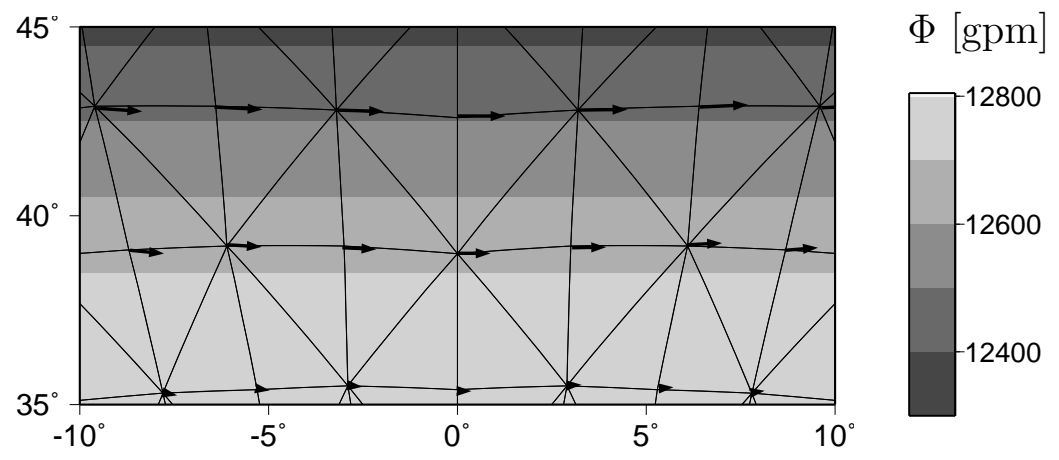

Figure 10: Unsteady jet stream, velocity $\mathbf{u}$, geopotential $\Phi, \Delta x=522 \mathrm{~km}$, section $\left(-10^{\circ}, 10^{\circ}\right) \times\left(35^{\circ}, 45^{\circ}\right)$ 
Preprint 2005, Alfred Wegener Institute, Germany, M. Läuter, D. Handorf, K. Dethloff

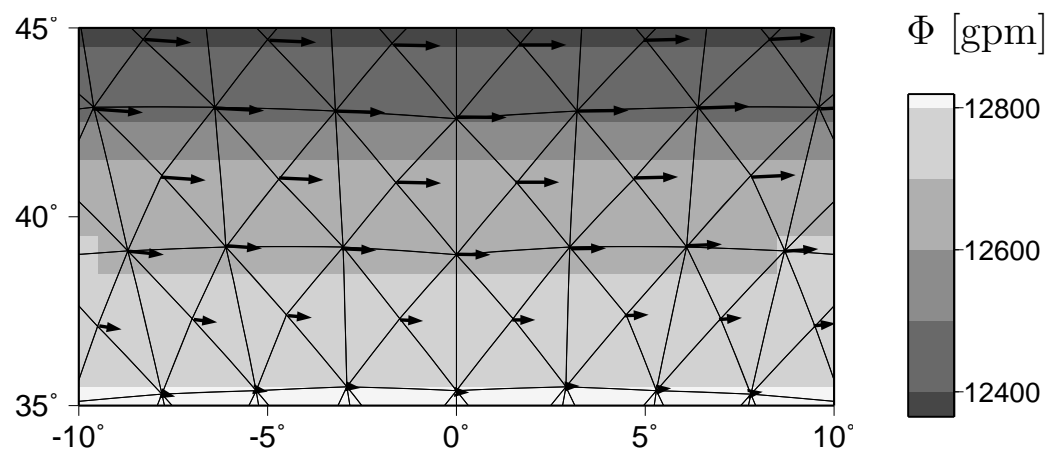

Figure 11: Unsteady jet stream, velocity $\mathbf{u}$, geopotential $\Phi, \Delta x=428 \mathrm{~km}$, section $\left(-10^{\circ}, 10^{\circ}\right) \times\left(35^{\circ}, 45^{\circ}\right)$ 